\title{
Optima localization by vehicle formations imitating the Nelder-Mead simplex algorithm
}

\author{
Shahab Kalantar • Uwe R. Zimmer
}

Received: 6 February 2008 / Accepted: 10 June 2009 / Published online: 20 August 2009

(C) Springer Science+Business Media, LLC 2009

\begin{abstract}
In this paper, we address the problem of localizing extrema points and iso-contours of ambient environmental fields (specifically, ocean bottom landscape and underwater plumes) using a networked formation of autonomous underwater vehicles. We propose the use of the Nelder-Mead extension to the basic simplex nonlinear optimization algorithm. In these robust gradient-free strategies, decisions are solely made based on field values measured by the individual vehicles, while measurements are fused and actions decided according to the algorithm. A main goal of this paper is to trigger interest in direct search methods as pertains to this type of robotic problem.
\end{abstract}

Keywords Distributed control · Swarm · Fomation · Localization $\cdot$ Nelder-Mead $\cdot$ Simplex

\section{Introduction}

This paper deals with navigation strategies for formations of autonomous underwater vehicles for the purpose of collectively localizing optima or iso-contours of an ambient underwater field (such as salinity or heat) or bottom terrain. This problem has been of increasing interest in the past few years. Such robotic systems can potentially have many useful applications. Locating deepest or highest points on a natural

Part of this research was done under a grant from Cooperative Research Centre for Intelligent Manufacturing Systems and Technology (CRC-IMST), Australia.

S. Kalantar $(\bowtie) \cdot$ U.R. Zimmer

College of Engineering and Computer Science, Australian

National University, Canberra, ACT 0200, Australia

e-mail: shahab.kalantar@rsise.anu.edu.au

U.R. Zimmer

e-mail: uwe.zimmer@ieee.org landscape, locating heat sources, and finding boundaries of a pollutant plume, are promising candidates.

Development in this area closely mimics that of function optimization theory, where optima of an arbitrary function are sought. Here, though, the environmental field plays the role of the function. The problem is usually considered in two dimensions. Some fields are intrinsically two dimensional (for example, bottom terrain). For three-dimensional phenomena (usually the result of some diffusion process), their projection on a horizontal plane is considered. Robots move constrained to this plane and sample the field.

Previous approaches: Mathematically speaking, if the field is smooth, the local gradient is the only information needed by a single robot to ascend or descend on the field. Guided by the gradient, the vehicle would always end up in a local optimum, depending on the case. The gradient is the main ingredient of a first-order approximation of the field. As such, the reached local optima may not be global ones. The method can be made more sophisticated by taking into account more terms of the Taylor series expansion which contain higher-order derivatives of the field (such as the Hessian). Derivative-based methods are the backbone of traditional optimization theory. Naturally enough, some robotics researchers have been prompted to use these techniques in applications where an environmental field plays the role of an objective function. The basic idea is to model the field around a local optimum $q_{0}$ by the quadratic (second order) approximation

$$
\begin{aligned}
F(q) & \approx \hat{F}(g) \\
& =\hat{c}+\hat{g}^{T}\left(q-q_{0}\right)+(1 / 2)\left(q-q_{0}\right)^{T} \hat{H}\left(q-q_{0}\right)+\varepsilon
\end{aligned}
$$

where $\hat{g}$ is an estimate of the gradient $\nabla F\left(q_{0}\right), \hat{H}$ is an estimate of the Hessian $\nabla^{2} F\left(q_{0}\right), \hat{c}$ is an estimate of $F\left(q_{0}\right)$, 
and $\varepsilon$ represents higher-order terms (assumed negligible). If a first-order approximation is deemed sufficient, a single robot, capable of estimating the gradient on its own, can use the control rule $\dot{q}(t)=-\hat{g}$. If the Hessian can also be estimated, the control rule would be $\dot{q}(t)=-\hat{H}^{-1} \hat{g}$ (see Robinet 2004). In practice, a single robot should visit a number of points, sample them and then employ some technique to estimate the gradient and/or the Hessian (see Pang et al. 2003 for an example). The parameters of the model are computed as functions of geometric relationships between sample points. In a multi-robot system, this process can be accelerated. In Byrne et al. (2003), robots in an aggregate home in on a source using least squares methods for estimating parameters of a quadratic model. In Ögren et al. (2002), triangular formations (using finite difference for gradient estimation) and square-shaped formations (using centred finite differences) are discussed. In Ögren et al. (2004), more general formations are considered using least squares methods for estimating the gradient. To reduce estimation error in methods based on first-order approximations, the shape of the formation (i.e., the degrees of freedom in a multi-robot system) has to be adjusted (Zhang and Leonard 2005; Ögren et al. 2002, 2004). Expressions for optimal inter-robot distances involve entries of the Hessian. The basic idea is to compute formation parameters by minimizing the statistical functional $E\left[(\boldsymbol{x}-\hat{\boldsymbol{x}})^{T}(\boldsymbol{x}-\hat{\boldsymbol{x}})\right]$ where $\boldsymbol{x}=\left(F\left(q_{0}\right), \nabla F\left(q_{0}\right)\right)$ and $\hat{\boldsymbol{x}}=(\hat{c}, \hat{g})$. Additionally, history of measurements and an iterative estimation scheme (such as the Kalman filter) can be used to improve estimation (Ögren et al. 2004).

As opposed to gradient-based methods, zero-order methods do not rely on the estimation of derivatives of the objective function. These methods can roughly be classified as derivative-free methods which approximate the objective function without using Taylor series expansions and direct search methods which only require the relative ranks of a countable set of function values (for unconstrained optimization). There is a lot of confusion in the relevant literature about this nomenclature. The above definition of a direct search is taken from Lewis et al. (2000). Examples of derivative-free methods include trust-region methods which use linear or quadratic models, assumed to be valid in a region around the current iterate. Optima of the local model is chosen as the next iterate. Examples of direct search methods include the simplex method, the Nelder-Mead modified simplex method, and pattern search methods. Unlike gradient-based methods, in which the current solution is updated by a differential inclusion (thus moving continuously), the current solution (iterate) in a zero-order method jumps from one iteration to the next. In a formation employing a zero-order method, one robot in the aggregate represents the current iterate and some of the robots visit a special pattern of positions. A comparison of values of the field at these positions determines one of the positions already occupied or visited by a robot as the next iterate. Gradient-free methods are used when the objective function is too noisy and it is meaningless to even talk about gradients. This is the main characteristic of natural fields. Direct search techniques fell out of favour in the optimization community for decades because they were based on seemingly ad-hoc rules. Only recently, convergence results have been discovered for them, putting them back in favour again (Kolda et al. 2003; McKinnon 1998; Lagarias et al. 1998; Wright 1996; Conn et al. 1997). As early as 1996, the authors of (Burian et al. 1996) propose the use of the simplex method as one which is especially suited to underwater applications. In Silva et al. (2004), de Sousa et al. (2005, 2006), and Speranzon et al. (2004), formation coordination strategies based on the simplest form of simplex search are proposed. In Kalantar and Zimmer (2007a, 2007b), the simple simplex method has been extended to incorporate the capability to traverse isocontours. The strength of the simplex approach stems from the fact that only relative values are used and not the exact ones.

Proposed approach: In this paper, we take this a step further by considering the modified versions of the simplex search which is superior to the basic simplex strategy. However, our aim in this paper is not to propose particular strategies but rather to emphasize the general underlying structure of zero order methods and arrive at a general framework that can be used for modelling and coordinating the class of aggregates which employ these methods. This paper is a first step towards this goal. Zero-order methods are very robust and, in most cases, converge very quickly to a vicinity of optima. However, they are not generally suitable for fine tuning. For this purpose, some researchers have proposed combining the two main strategies and switch to a Newton-type algorithm when close enough to optima. We do not pursue this possibility here. Refer to Kolda et al. (2003) for detailed exposition of recent trends in this area.

The proposed strategy is based on the Nelder-Mead version of the simplex optimization method. In this gradientfree method, decisions are solely made based on field values measured by the individual vehicles, while measurements are fused according to the algorithm. Unlike the basic simplex method, Nelder-Mead algorithm acts aggressively, trying to explore longer distances if there is any chance of improvement. We make several modifications. Some have already been introduced in the optimization literature. Simplex gradients are used to avoid immature stagnation. Constrains are enforced to prevent the formation from getting too much deformed or getting too small. The $\alpha$-constrained method is used to constrain the formation to certain areas and avoid obstacles. Several other relevant modifications are discussed, as well convergence results. Some issues particular to implementation on robots in the real world, as well as globalization of the search, are elaborated on. The simplex algorithm is a discrete process. As such, hybrid automata 
Fig. 1 Vehicles and networks, (a) Serafina. (b) Three-vehicle formation immersed in a field (top view)

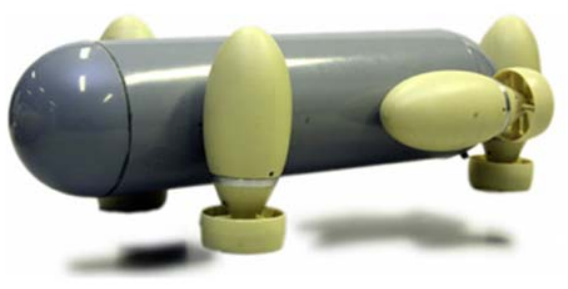

(a) (b) can be employed as appropriate tools for modelling and controller design. The hybrid state automaton introduced here plays the role of the virtual formation controller. Of particular interest here is that unlike most robotic formation applications, those which implement direct search methods cannot be rigid (Eren 2004) as the formation itself becomes part of the search algorithm and thus call for a more general strategy for formation control.

Physical setups: The strategies discussed here is not targeted towards any specific vehicle. Any underwater vehicle with the following set of generic capabilities could be a candidate:

1. Communication with peer robots in the network. This capability is crucial to synchronization of actions. Long wave radio is a potential means.

2. Measurement of range and bearing to other robots. A synchronized combination of optical and ultra-sound sensor modules can be used for this purpose.

3. Global orientation sensing (using a compass module). This is used for calculating the heading of a robot.

4. Ambient water flow sensing for keeping station.

5. Depth sensing (using pressure sensors). We assume that the vehicles move on a horizontal plane parallel to the surface of the water.

6. Field sensing. The type of sensor is application specific. Examples include temperature and salinity sensors, and sonar for underwater landscape.

7. GPS sensing when resurfacing.

Figure 1 shows a Serafina robot and a network consisting of three Serafinas (see http://users.rsise.anu.edu.au/ serafina/ for more detail). While moving on the plane, robots can be controlled to go to desired positions expressed relative to positions and headings of other reference robots. The lowlevel control system is composed of two sub-controllers: (1) the horizontal controller produces torques for the horizontal thrusters for moving on a plane, and (2) the vertical controller produces torques for vertical thrusters for stabilization on the desired plane of motion. In this paper, we will not concern ourselves with any of the above low-level issues as we focus here on planning.

Paper organization: We begin, in Sect. 2, by stating the basic Nelder-Mead simplex algorithm, along with introducing the relevant notations and definitions. Following that, in Sect. 3, we will some convergence properties of the basic algorithm. Section 4 is devoted to a short account of numerous modifications of and improvements to the basic version which have been reported in the literature. The shape constraint and necessary modifications are discussed in Sect. 5. The modified algorithm is stated in Sect. 6. In Sect. 7, actual implementation on robotic platforms is discussed. Finally, simulation results are presented in Sect. 8 to demonstrate the algorithm.

\section{Basic Nelder-Mead algorithm}

The Nelder-Mead algorithm (Nelder and Mead 1965) belongs to the class of direct search algorithms which do not require the knowledge of the gradient. It is primarily used for the unconstrained minimization of an objective function $f$ which can be nonconvex, discontinuous or noisy. In the planar case, the problem is defined as

$\min _{q \in \mathfrak{R}^{2}} f(q)$.

We use the notation $S\left[q_{i}, q_{2}, q_{3}\right]$ to represent a planar simplex with respective vertices. The algorithm starts with an 
initial simplex $S^{(0)}$, composed of three points (vertices) $q_{1}^{(0)}, q_{2}^{(0)}$, and $q_{3}^{(0)}$ in the plane. The simplex is then iteratively changed to generate the sequence $S^{(k)}$ of simplexes. We use the notation $q^{(k)}=\left(q_{1}^{(k)}, q_{2}^{(k)}, q_{3}^{(k)}\right)^{T}$ to denote the positional state of the algorithm. The change to positions of the vertices of $S^{(k)}$ are made based on the values $f\left(q_{1}^{(k)}\right), f\left(q_{2}^{(k)}\right)$, and $f\left(q_{3}^{(k)}\right)$, which leads to the generation of $S^{(k+1)}$. The algorithm terminates if some function $T$ of the values at the vertices satisfies an inequality of the general form $T\left(q^{(k)}\right) \leq \varepsilon$. It is required that $S^{(0)}$ be non-degenerate, i.e., the vectors $q_{i}^{(k)}-q_{j}^{(k)}, i, j=1,2,3, i \neq j$, be linearly independent. The algorithm uses four given numbers $\delta_{r}$ (reflection coefficient), $\delta_{2}$ (expansion coefficient), $\delta_{1}$ (outside contraction coefficient), and $\delta_{-1}$ (inside contraction coefficient), which are required to satisfy the relation

$-1<\delta_{-1}<0<\delta_{1}<\delta_{r}<\delta_{2}$.

Below, we state a formal version of the algorithm.

Algorithm 1 Nelder-Mead Simplex $\left(S^{(0)}, f, \varepsilon\right)$ Set $k=0$ and go to step 1-1.

\section{Step 1-1 (Sort the vertices)}

If $T\left(S^{(k)}\right) \leq \varepsilon$, exit. Label the vertices, arbitrarily, as $q_{1}^{(k)}$, $q_{2}^{(k)}$ and $q_{3}^{(k)}$. Sort the vertices of the current simplex $S^{(k)}$ in ascending order of function values, producing a re-labelled version of the vertices

$q_{w}^{(k)}=\underset{q_{i}^{(k)}}{\operatorname{argmin}}\left\{f\left(q_{i}^{(k)}\right)\right\}$,

$q_{b}^{(k)}=\underset{q_{i}^{(k)}}{\operatorname{argmax}}\left\{f\left(q_{i}^{(k)}\right)\right\}$,

$q_{s}^{(k)}=\underset{q_{i}^{(k)}, q_{i}^{(k)} \neq q_{b}^{(k)}}{\operatorname{argmax}}\left\{f\left(q_{i}^{(k)}\right)\right\}$.

Thus, we have $f\left(q_{w}^{(k)}\right) \leq f\left(q_{s}^{(k)}\right) \leq f\left(q_{b}^{(k)}\right) . q_{w}^{(k)}, q_{b}^{(k)}$, and $q_{s}^{(k)}$, are called, respectively, the worst, the best, and the second (or next) best (or worst) vertices. Compute the centroid

$\bar{q}^{(k)}=\frac{1}{2}\left(q_{s}^{(k)}+q_{b}^{(k)}\right)$

of

$S^{(k)} \backslash\left\{q_{w}^{(k)}\right\}$.

Denote

$z^{(k)}\left(\delta_{r}\right)=\left(1+\delta_{r}\right) \bar{q}^{(k)}-\delta q_{w}^{(k)}$,

and

$S_{\delta}^{(k)}=\left(S^{(k)} \backslash\left\{q_{w}^{(k)}\right\}\right) \cup\left\{z^{(k)}\left(\delta_{r}\right)\right\}$

(i.e., $S_{\delta}^{(k)}=S\left[q_{1}^{(k)}, q_{2}^{(k)}, z^{(k)}\left(\delta_{r}\right)\right]$ ).
Step 1-2 (Reflection)

If

$f\left(q_{b}^{(k)}\right) \leq f\left(z^{(k)}\left(\delta_{r}\right)\right)$

and

$f\left(z^{(k)}\left(\delta_{r}\right)\right)<f\left(q_{s}^{(k)}\right)$,

set $S^{(k+1)}=S_{\delta_{r}}^{(k)}\left(\right.$ replace $q_{w}^{(k)}$ with $\left.z^{(k)}\left(\delta_{r}\right)\right)$ and go to step $1-1$.

Step 1-3 (Expansion)

If $f\left(z^{(k)}\left(\delta_{r}\right)\right) \geq f\left(q_{b}^{(k)}\right)$, go to step 1-4.

If

$f\left(z^{(k)}\left(\delta_{r}\right)\right)<f\left(q_{b}^{(k)}\right)$

and

$f\left(z^{(k)}\left(\delta_{2}\right)\right)<f\left(z^{(k)}\left(\delta_{r}\right)\right)$,

set $S^{(k+1)}=S_{\delta_{2}}^{(k)}$. Otherwise, set $S^{(k+1)}=S_{\delta_{r}}^{(k)}$. Go to step $1-1$.

Step 1-4 (Outside contraction)

If

$f\left(q_{s}^{(k)}\right)>f\left(z^{(k)}\left(\delta_{r}\right)\right)$

or

$f\left(z^{(k)}\left(\delta_{r}\right)\right) \geq f\left(q_{w}^{(k)}\right)$,

go to step 1-5.

If

$f\left(q_{s}^{(k)}\right) \leq f\left(z^{(k)}\left(\delta_{r}\right)\right)$

and

$f\left(z^{(k)}\left(\delta_{r}\right)\right)<f\left(q_{w}^{(k)}\right)$

and

$f\left(z^{(k)}\left(\delta_{1}\right)\right)<f\left(q_{w}^{(k)}\right)$,

set $S^{(k+1)}=S_{\delta_{1}}^{(k)}$ and go to step 1-1.

Otherwise, go to step 1-6.

Step 1-5 (Inside contraction)

If $f\left(z^{(k)}\left(\delta_{r}\right)\right)<f\left(q_{w}^{(k)}\right)$, go to step 1-6.

If

$f\left(z^{(k)}\left(\delta_{r}\right)\right) \geq f\left(q_{w}^{(k)}\right)$

and

$f\left(z^{(k)}\left(\delta_{-1}\right)\right)<f\left(q_{w}^{(k)}\right)$,

set $S^{(k+1)}=S_{\delta_{-1}}^{(k)}$ and go to step 1-1. 
Otherwise go to step 1-6.

\section{Step 1-6 (Shrink)}

Set

$S^{(k+1)}=q_{b}^{(k)}+\frac{1}{\xi}\left(S^{(k)}-q_{b}^{(k)}\right)$

and go to step 1-1.

In the algorithm, $\xi>0$ determines how much the simplex should reduce in size and is usually taken to be $\xi=2$. To sort the vertices, any tie-breaking strategy can be used. In essence, the algorithm, just like the original simplex, tries to replace the worst vertex with a new better one. In any case, the average value

$\overline{f\left(q^{(k)}\right)}=\frac{1}{3} \sum_{i=1}^{3} f\left(q_{i}^{(k)}\right)$

is decreased. The value state of $S^{(k)}$ can either be defined as $f\left(q_{b}^{(k)}\right)$ or $\overline{f\left(q^{(k)}\right)}$. The success of the Nelder-Mead algorithm lies in the fact that it allows non-isometric reflections. This same feature also makes this method problematic if certain modifications are not considered, as the next section will show. The values usually used for $\delta$ parameters are $\delta_{r}=1$, $\delta_{2}=2, \delta_{1}=1$, and $\delta_{-1}=-1 / 2$.

One popular choice for $T$ is

$T\left(S^{(k)}\right)=\left(\frac{1}{3} \sum_{i=1}^{3}\left(f\left(q_{i}^{(k)}\right)-\overline{f\left(q^{(k)}\right)}\right)^{2}\right)^{1 / 2}$.

Another choice is simply

$T\left(S^{(k)}\right)=\left|f\left(q_{w}^{(k)}\right)-f\left(q_{b}^{(k)}\right)\right|$.

These termination criteria test for flatness of the simplex. Other choices will be discussed in the next section. There are other termination criteria which relate to the size of the simplex rather than function values. Among these is smallness (Luersen and Le Riche 2004)

$\max _{v \in\left\{v_{12}, v_{13}, v_{23}\right\}}\left(\frac{\left\langle v, e_{x}\right\rangle}{X_{+}-X_{-}}+\frac{\left\langle v, e_{y}\right\rangle}{Y_{+}-Y_{-}}\right)<\varepsilon$,

where $v_{i j}=q_{j}^{(k)}-q_{i}^{(k)}$ denote the edges of the simplex, $e_{x}$ and $e_{y}$ are unit vectors in the directions of the global coordinate system, and $X_{+}, X_{-}, Y_{+}$and $Y_{-}$are some bounds. Degeneracy can also be used to determine if the simplex has been stuck in a subspace from which it cannot escape (Luersen and Le Riche 2004). A simplex is degenerate if it is not small but either

$$
\frac{\min _{v \in\left\{v_{12}, v_{13}, v_{23}\right\}}\|v\|}{\max _{v \in\left\{v_{12}, v_{13}, v_{23}\right\}}\|v\|}<\varepsilon
$$

or

$\frac{\operatorname{det}\left(\left[v_{12}, v_{13}, v_{23}\right]\right)}{\left\|v_{12}\right\|\left\|v_{13}\right\|\left\|v_{23}\right\|}<\varepsilon$.

The rationale behind reflection is that the function value decreases along the side of the simplex triangle from $q_{w}^{(k)}$ to $q_{b}^{(k)}$. This is also true for the side from $q_{w}^{(k)}$ to $q_{s}^{(k)}$. Hence, it is possible that $f(q)$ takes on smaller values at points which lie away from $q_{w}^{(k)}$ on the opposite side of the good side (the line connecting $q_{b}^{(k)}$ to $q_{s}^{(k)}$ ). Figure 2 shows the different operations. The reflection operation is based on this simple observation (Fig. 2(b)). If the reflected point $q_{r}^{(k)}=z^{(k)}\left(\delta_{r}\right)$ gives indeed a better value, then the direction towards the minimum is correct and it might be the case that if we go even farther (to $q_{e}^{(k)}=z^{(k)}\left(\delta_{2}\right)$ ), we get an even better value, which is the reasoning behind the expansion operation (Fig. 2(c)). If the reflection does not provide a better solution, then we should move backwards and try two points which are located on the line connecting $q_{w}^{(k)}$ and $q_{r}^{(k)}$. These points are $q_{o c}^{(k)}=z^{(k)}\left(\delta_{1}\right)$ and $q_{i c}^{(k)}=z^{(k)}\left(\delta_{-1}\right)$ (Fig. 2(d) and Fig. 2(e)). If this also fails, we have no choice but to shrink the simplex towards the best vertex (Fig. 2(f)).

In the literature, it is the convention to set $w=3, s=2$, and $b=1$, such that we always have $f\left(q_{1}^{(k)}\right) \leq f\left(q_{2}^{(k)}\right) \leq$ $f\left(q_{3}^{(k)}\right)$. We will follow this convention from now on.

\section{Properties}

Here, we review some of the properties of the basic algorithm. First, we introduce some useful definitions.

\section{Definition 1}

1. $V\left(S^{(k)}\right)$ denotes the $2 \times 2$ matrix of simplex directions defined by

$V\left(S^{(k)}\right)=\left(q_{2}^{(k)}-q_{1}^{(k)}, q_{3}^{(k)}-q_{1}^{(k)}\right)$.

For a nondegenerate simplex, $V\left(S^{(k)}\right)$ is nonsingular.

2. The outer diameter of the simplex is defined by

$\operatorname{diam}_{o}\left(S^{(k)}\right)=\max _{i, j \in\{1,2,3\}}\left\|q_{i}^{(k)}-q_{j}^{(k)}\right\|$.

3. The inner diameter is defined as

$\operatorname{diam}_{i}\left(S^{(k)}\right)=\min _{i, j \in\{1,2,3\}}\left\|q_{i}^{(k)}-q_{j}^{(k)}\right\|$.

4. The volume of a simplex is defined as

$\operatorname{vol}\left(S^{(k)}\right)=\frac{1}{6}\left\|\operatorname{det}\left(V\left(S^{(k)}\right)\right)\right\|$.

Thus, a simplex is nondegenerate if $\operatorname{vol}\left(S^{(k)}\right)>0$. 
Fig. 2 Operations of the

Nelder-Mead simplex method with vehicles playing the role of simplex vertices. (a) Initial simplex. (b) Reflection. (c) Expansion. (d) Inside contraction. (e) Outside contraction. (f) Shrink
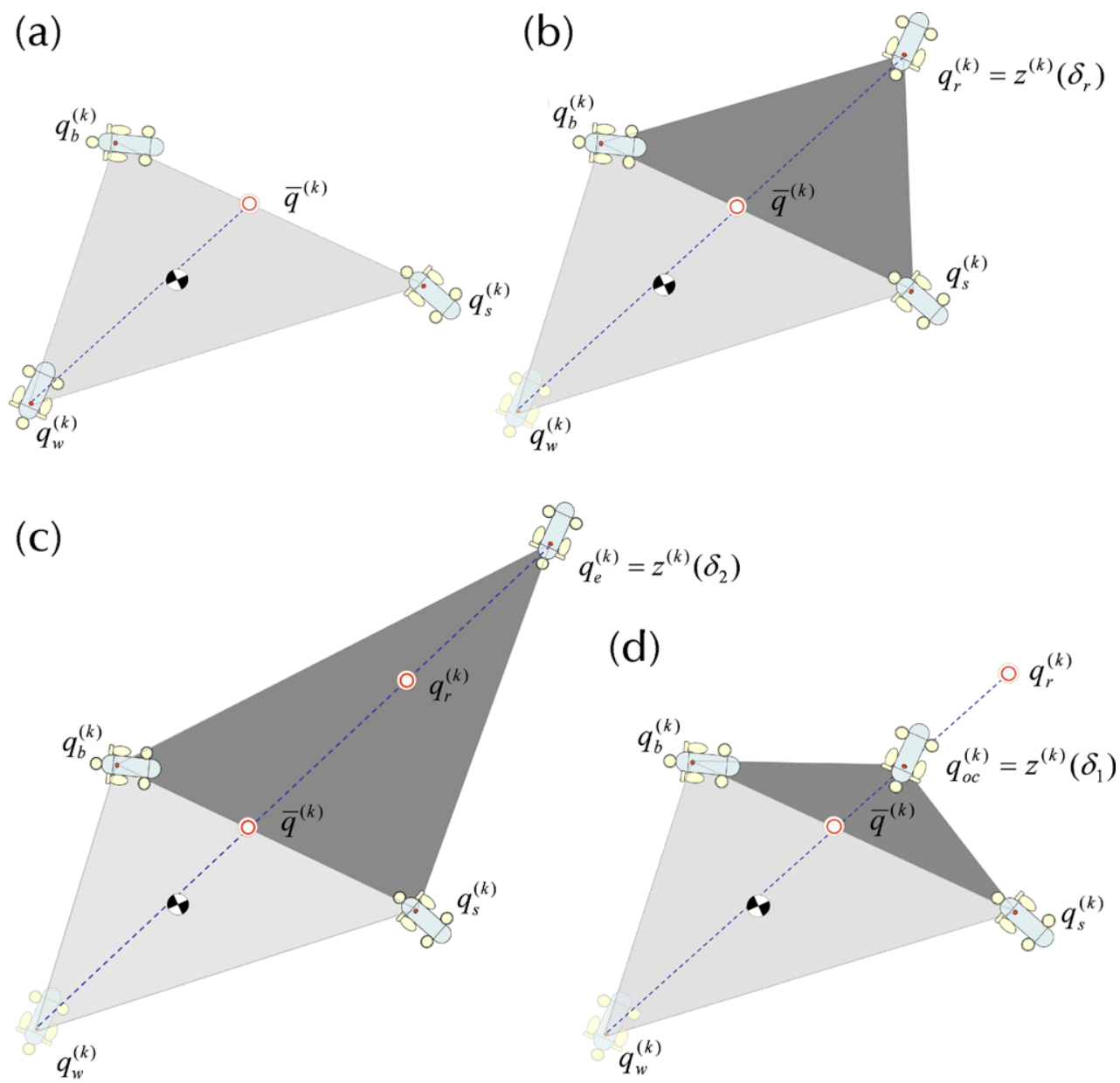

(e)
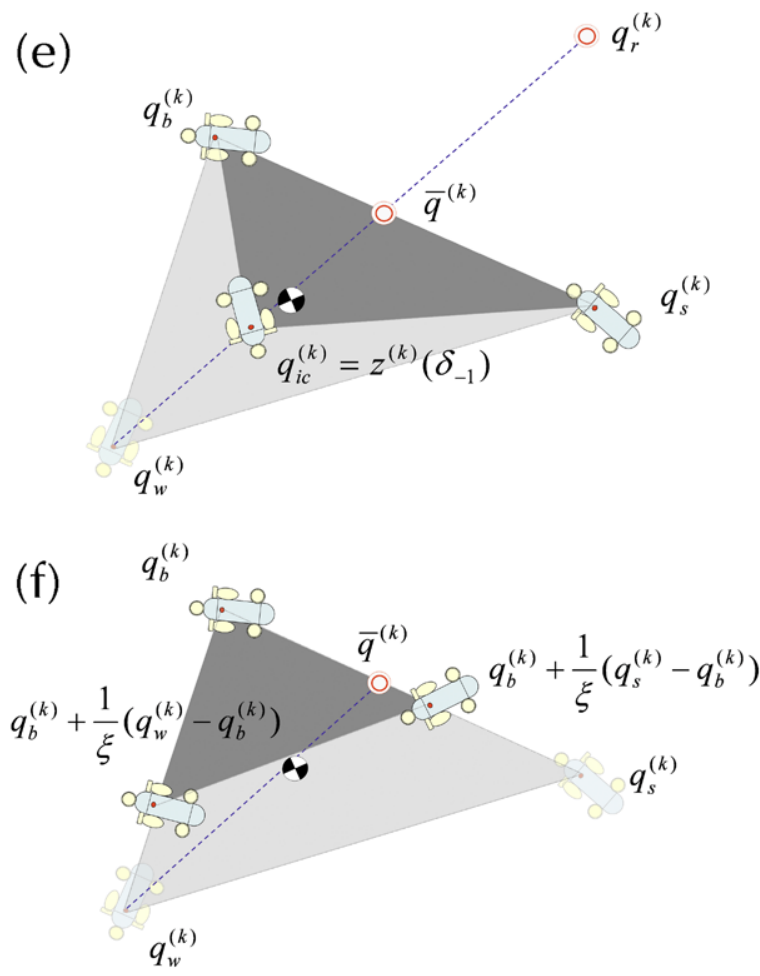
5. If, in a non-shrink step, the coefficient $\tau \in\left\{\delta_{r}, \delta_{2}, \delta_{1}\right.$, $\left.\delta_{-1}\right\}$ is used to generate the next simplex, we call that step of type $\tau$.

Now, we state some properties. See (Lagarias et al. 1998) for proofs and further discussion.

\section{Lemma 1}

1. If the initial simplex $S^{(0)}$ is nondegenerate, then so are all the other subsequent simplexes.

2. Following a non-shrink step of type $\tau$,

$$
\operatorname{vol}\left(S^{(k+1)}\right)=|\tau| \operatorname{vol}\left(S^{(k)}\right) \text {. }
$$

3. Following a shrink step,

$$
\operatorname{vol}\left(S^{(k+1)}\right)=\frac{1}{\xi^{2}} \operatorname{vol}\left(S^{(k)}\right) .
$$

4. The Nelder-Mead method is invariant under affine transformation $\phi(q)=\boldsymbol{A} q+\boldsymbol{b}, \boldsymbol{A}$ invertible. When minimizing $f(q)$, starting with simplex $S^{(0)}$, the complete sequence of Nelder-Mead steps and function values is the same as when minimizing the function $\tilde{f}(q)=f(\phi(q))$ with initial simplex $\tilde{S}^{(0)}$ defined by

$$
\tilde{S}^{(0)}=\phi^{-1}\left(S^{(0)}\right)=\boldsymbol{A}^{-1}\left(S^{(0)}\right)-\boldsymbol{A}^{-1} \boldsymbol{b} .
$$

5. The sequence $\left\{f_{1}^{(k)}\right\}$ always converges.

6. At every nonshrink iteration, $f_{i}^{(k+1)} \leq f_{i}^{(k)}, i \in\{1,2,3\}$.

7. If there is only a finite number of shrink operations, then each sequence $\left\{f_{i}^{(k)}\right\}$ converges as $k \rightarrow \infty, f_{i}^{\infty} \leq f_{i}^{(k)}$, and $f_{1}^{\infty} \leq f_{2}^{\infty} \leq f_{3}^{\infty}$, where $f_{i}^{\infty}=\lim _{k \rightarrow \infty} f_{i}^{(k)}$.

8. If there are only a finite number of nonshrink iterations, then all simplex vertices converge to a single point.

9. If is strictly convex on $\mathfrak{R}^{2}$ and the algorithm starts with a nondegenerate initial simplex, then no shrink steps will be taken.

The next lemma summarizes some important convergence properties for the planar case for strictly convex functions with bounded level sets. $f$ is strictly convex on $\mathfrak{R}^{2}$ if, for every pair of points $q_{1}$ and $q_{2}, q_{1} \neq q_{2}$, and every $0<\lambda<1$, we have

$f\left(\lambda q_{1}+(1+\lambda) q_{2}\right)<\lambda f\left(q_{1}\right)+(1+\lambda) f\left(q_{2}\right)$.

Also, the level set $\Gamma_{\mu}(f)$ is defined as $\Gamma_{\mu}(f)=\{q:(f(q) \leq$ $\mu)\} . f$ has bounded level sets if $\Gamma_{\mu}(f)$ is bounded for every $\mu$. A strictly convex function with bounded level sets has a unique minimizer.

Lemma 2 Let $f$ be a strictly convex function on $\mathfrak{R}^{2}$ with bounded level sets, the algorithm starts with a nondegenerate simplex $S^{(0)}$, and $\delta_{r}=1, \delta_{2}=2, \delta_{1}=1 / 2$, and $\delta_{-1}=$ $-1 / 2$.
1. $f_{1}^{\infty}=f_{2}^{\infty}=f_{3}^{\infty}$.

2. If the best vertex $q_{1}^{(k)}$ is constant for all $k$, then the simplices $S^{(k)}$ converge to $q_{1}^{(0)}$ as $k \rightarrow \infty$.

3. The simplices $\left\{S^{(k)}\right\}$ generated by the algorithm satisfy $\lim _{k \rightarrow \infty} \operatorname{vol}\left(S^{(k)}\right)=0$.

4. We also have $\lim _{k \rightarrow \infty} \operatorname{diam}_{o}\left(S^{(k)}\right)=0$.

\section{Variations}

It is well-known that the Nelder-Mead method can stagnate and converge to non-optimal points even for very simple functions. In McKinnon (1998), a family of examples are given for which the algorithm converges to a non-stationary point. To remedy this, many variations of the original algorithm have been proposed. In this section, we discuss some of them as well as other modifications which relate to globalization of the algorithm and those that make the algorithm more suitable for implementation by robotic vehicles. We first give some definitions which will be used.

\section{Definition 2}

1. The simplex condition $\kappa\left(S^{(k)}\right)$ is defined as the $l^{2}$ condition number of $V\left(S^{(k)}\right) . \delta\left(f: S^{(k)}\right)$ denotes the vector of objective function differences

$$
\delta\left(f: S^{(k)}\right)=\left(f\left(q_{2}^{(k)}\right)-f\left(q_{1}^{(k)}\right), f\left(q_{3}^{(k)}\right)-f\left(q_{1}^{(k)}\right)\right)^{T} .
$$

2. Oriented lengths of the simplex are defined as

$$
\begin{aligned}
& \sigma_{+}\left(S^{(k)}\right)=\max _{j \in\{1,2,3\}}\left\|q_{1}^{(k)}-q_{j}^{(k)}\right\|, \\
& \sigma_{-}\left(S^{(k)}\right)=\min _{j \in\{1,2,3\}}\left\|q_{1}^{(k)}-q_{j}^{(k)}\right\| .
\end{aligned}
$$

We have $\sigma_{+}\left(S^{(k)}\right) \leq \operatorname{diam}_{o}\left(S^{(k)}\right) \leq \sigma_{-}\left(S^{(k)}\right)$.

3. Define

$$
v\left(S^{(k)}\right)=\frac{\left|\operatorname{det}\left(V\left(S^{(k)}\right)\right)\right|}{\operatorname{diam}_{o}\left(S^{(k)}\right)^{2}} .
$$

$v\left(S^{(k)}\right)=0$ if one of the interior angles of $S^{(k)}$ is zero.

Simplex gradients and sufficient decrease: In Kelley (1999), a very useful variant of the algorithm is proposed which we will adopt here. They based their method on the omission of the shrink step altogether, arguing that it very rarely occurs in practice. They, instead, add an additional step which they call oriented restart. It is very similar to the shrink operation but the directions are a function of the simplex gradient. 
Definition 3 The simplex gradient $D\left(f: S^{(k)}\right)$ is defined as

$D\left(f: S^{(k)}\right)=V\left(S^{(k)}\right)^{-T} \delta\left(f: S^{(k)}\right)$.

They consider functions of the form $f(q)=g(q)+\zeta(q)$, where $g(q)$ is a smooth function and $\zeta(q)$ is a low-amplitude perturbation.

\section{Lemma 3}

1. Let $\nabla f$ be Lipschitz continuous in a neighborhood of $S^{(k)}$ with Lipschitz constant $2 K_{f}$. Then there exists $K>$ 0 , depending only on $K_{f}$, such that

$$
\left\|\nabla f\left(q_{1}^{(k)}\right)-D\left(f: S^{(k)}\right)\right\| \leq K \kappa\left(V\left(S^{(k)}\right)\right) \sigma_{+}\left(S^{(k)}\right) .
$$

2. Suppose $f=g+\zeta$. Let $\nabla g$ be Lipschitz continuous in a neighborhood of $S^{(k)}$ with Lipschitz constant $2 K_{g}$. Then there exists $K>0$, depending only on $K_{g}$, such that

$$
\begin{aligned}
& \left\|\nabla g\left(q_{1}^{(k)}\right)-D\left(f: S^{(k)}\right)\right\| \\
& \quad \leq K \kappa\left(V\left(S^{(k)}\right)\right)\left(\sigma_{+}\left(S^{(k)}\right)+\frac{\|\zeta\|_{S^{(k)}}}{\sigma_{+}\left(S^{(k)}\right)}\right) .
\end{aligned}
$$

\section{Assumption 1}

1. $S^{(0)}$ is non-singular.

2. For each $k, \overline{f\left(q^{(k+1)}\right)}<\overline{f\left(q^{(k)}\right)}$.

Their method is based on requiring that the $(k+1)$-th iteration satisfy

$\overline{f\left(q^{(k+1)}\right)}-\overline{f\left(q^{(k)}\right)}<-\alpha\left\|D\left(f: S^{(k)}\right)\right\|^{2}$,

where

$\alpha=\alpha_{0} \frac{\sigma_{+}\left(S^{(0)}\right)}{\left\|D\left(f: S^{(0)}\right)\right\|}$

and typically $\alpha_{0}=10^{-4}$.

\section{Theorem 1}

1. Let the assumptions Assumption 1, as well as the assumptions of Lemma 3(1) hold true, with the Lipschitz constant $K^{k}$ bounded. Assume that $\left\{\overline{f\left(q^{(k)}\right)}\right\}$ is bounded from below. Then, if (44) holds for all but finitely many $k$ and the product $\sigma_{+}\left(S^{(k)}\right) \kappa\left(V\left(S^{(k)}\right)\right) \rightarrow 0$, then any accumulation point of the simplices is a critical point of $f$.

2. Let Assumption 1 hold true, as well as the assumptions of Lemma 3(2), with the Lipschitz constants $K_{g}^{k}$ uniformly bounded. Assume that $\left\{\overline{f\left(q^{(k)}\right)}\right\}$ is bounded from below. Then, if (44) holds for all but finitely many $k$ and if

$$
\lim _{k \rightarrow \infty} \kappa\left(V\left(S^{(k)}\right)\right)\left(\sigma_{+}\left(S^{(k)}\right)+\frac{\|\zeta\|_{S^{(k)}}}{\sigma_{+}\left(S^{(k)}\right)}\right)=0,
$$

then any accumulation point of the simplices is a critical point of $g$. Furthermore, $\lim _{k \rightarrow \infty} D\left(f: S^{(k)}\right)=0$.

It is proposed in Kelley (1999) that whenever (44) fails but $\overline{f\left(q^{(k+1)}\right)}-\overline{f\left(q^{(k)}\right)}<0$, an oriented restart should be carried out by replacing the vertices with new $\tilde{q}_{1}=q_{1}$, $\tilde{q}_{2}=\tilde{q}_{1}+\beta_{1} e_{1}, \tilde{q}_{3}=\tilde{q}_{1}+\beta_{2} e_{2}$, where $e_{1}$ and $e_{2}$ are unit coordinate vectors and

$$
\begin{aligned}
& \beta_{l}=\frac{1}{2} \sigma_{-}\left(S^{(k)}\right) \operatorname{sign}\left(\left\langle D\left(f: S^{(k)}\right), e_{l}\right\rangle\right), \\
& \quad \text { if }\left\langle D\left(f: S^{(k)}\right), e_{l}\right\rangle \neq 0,
\end{aligned}
$$

and

$\beta_{l}=\frac{1}{2} \sigma_{-}\left(S^{(k)}\right), \quad$ if $\left\langle D\left(f: S^{(k)}\right), e_{l}\right\rangle=0$,

where $l \in\{1,2\}$. More compactly,

$\beta_{l}=\frac{1}{2} \sigma_{-}\left(S^{(k)}\right) \eta\left(\operatorname{sign}\left(\left\langle D\left(f: S^{(k)}\right), e_{l}\right\rangle\right)\right)$,

where $\eta(u)=\operatorname{sign}(u)$ if $u \neq 0$ and $\eta(u)=1$ otherwise. The diameter of the new simplex is $\sigma_{-}\left(S^{(k)}\right) / \sqrt{2}$. The new simplex has orthogonal edges and is a difference approximation to the steepest descent step from the current best point. To quote (Kelley 1999), this re-orientation is intended to compensate for a certain kind of stagnation in which the best vertex remains unchanged throughout the iteration and the simplices converge to that point. It is argued that, although the simplex gradient may not be a very good approximation of the true gradient, it contains enough information to determine an orthant to locate the new simplex. Note that only the sign is used. After several oriented restarts in a row, the algorithm should terminate. Refer to Kelley (1999) for a more detailed discussion and simulations.

Constrained optimization: The original Nelder-Mead algorithm, as well as the variants above, are all unconstrained. Here, we would like to optimize $f(q)$ subject to the constraint $g(q) \leq 0$ which defines a feasible region $\Omega^{s}$ inside the search space, which, in our case is $\mathfrak{R}^{2}$. The constraints are used to ensure that the simplex formation does not escape a certain region of exploration and also to avoid obstacles. Figure 3 shows a boundary $\gamma$ past which the formation should not go. The constraint can be defined as

$g(q)=\|Q(q, \gamma)-q\|-D$,

where $Q(q, \gamma)$ denotes the closest point on the boundary to $q$ and $D$ indicates a safe distance away from the boundary. In the case of a simplex formation, $q$ can be the position of any of the vertices or the centre of mass. The region defined by $g(q) \leq 0$ is the safe region, while $0<g(q) \leq D / 2$ defines the critical region $\Omega^{c}$. The region defined by $g(q)>$ $D / 2$ is the unsafe region $\Omega^{u}$. Many methods have been 


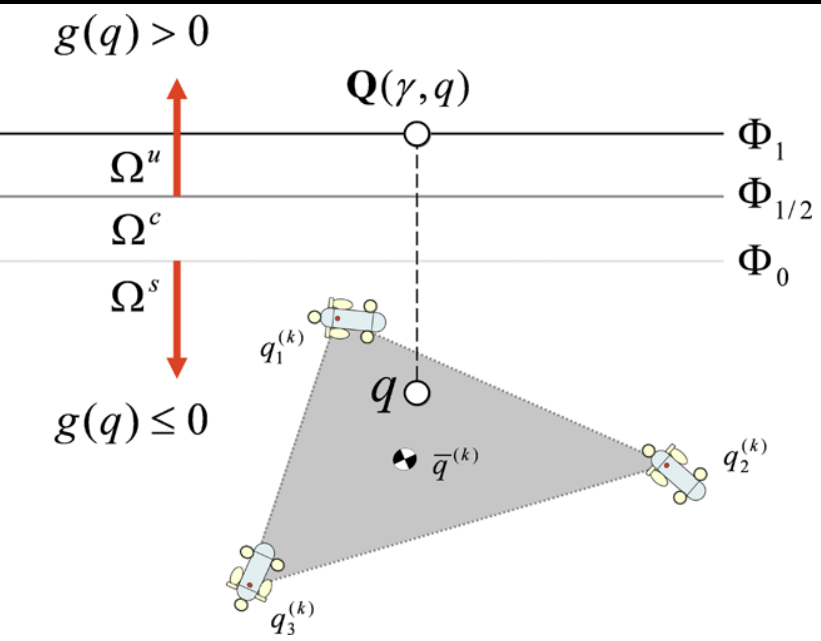

Fig. 3 Constraints. Boundary is the black line. $\Phi_{1}, \Phi_{1 / 2}$, and $\Phi_{0}$ represent, respectively, isocontours $g(q)=D, g(q)=D / 2$, and $g(q)=0$

proposed to transform an unconstrained optimization algorithm into a constrained one. Barrier methods add barrier terms to the objective function which grow to infinity as the point approaches the boundary of the constrained domain. Penalty methods add penalty terms to the objective function. Here, we adopt the version of the $\alpha$-constrained method proposed in Takahama and Sakai (2005). In this paper, the satisfaction level of a constraint for a point $q$ is defined by $\mu_{g}(q)=1$ if $g(q) \leq 0$ and $0 \leq \mu_{g}(q)<1$, otherwise. More specifically, we can define $\mu_{g}(q)=1-2 g(q) / D$, whenever $0 \leq g(q) \leq D / 2$.

They also define the $\alpha$-level comparison as an order relation on the set composed of pairs $\left(f(q), \mu_{g}(q)\right)$. The idea is that if the satisfaction level of a point is less than 1 , the point should be considered infeasible and its value should be low no matter what the function value $f(q)$ is. This makes $\mu_{g}(q)$ precede $f(q)$, as satisfying the constraint is more important than minimization. Given the pairs $\left(f\left(q_{1}\right), \mu_{g}\left(q_{1}\right)\right)$ and $\left(f\left(q_{2}\right), \mu_{g}\left(q_{2}\right)\right)$, then, for any $0 \leq \alpha \leq 1$, the $\alpha$-level comparisons $\leq_{\alpha}$ and $<_{\alpha}$ are defined as follows:

1. $\left(f\left(q_{1}\right), \mu_{g}\left(q_{1}\right)\right)<_{\alpha}\left(f\left(q_{2}\right), \mu_{g}\left(q_{2}\right)\right)$ if and only if $f\left(q_{1}\right)<f\left(q_{2}\right)$, when $\mu_{g}\left(q_{1}\right), \mu_{g}\left(q_{2}\right) \geq \alpha$ or $\mu_{g}\left(q_{1}\right)=$ $\mu_{g}\left(q_{2}\right)$, or $\mu_{g}\left(q_{1}\right)>\mu_{g}\left(q_{2}\right)$, otherwise.

2. $\left(f\left(q_{1}\right), \mu_{g}\left(q_{1}\right)\right) \leq_{\alpha}\left(f\left(q_{2}\right), \mu_{g}\left(q_{2}\right)\right)$ if and only if $f\left(q_{1}\right) \leq f\left(q_{2}\right)$, when $\mu_{g}\left(q_{1}\right), \mu_{g}\left(q_{2}\right) \geq \alpha$ or $\mu_{g}\left(q_{1}\right)=$ $\mu_{g}\left(q_{2}\right)$, or $\mu_{g}\left(q_{1}\right)>\mu_{g}\left(q_{2}\right)$, otherwise.

$\leq_{0}$ and $<_{0}$ are ordinary comparisons. $\leq_{1}$ and $<_{1}$ correspond to lexicographic order. Denote by $(P)$ the unconstrained minimization problem, by $\left(P_{\leq_{\alpha}}\right)$ the version of $(P)$ based on $\alpha$-level comparisons, and by $\left(P^{\alpha}\right)$ the minimization problem subject to $\mu_{g}(q) \geq \alpha$. The following results are proved in Takahama and Sakai (2005).

\section{Theorem 2}

1. If an optimal solution $\left(P^{1}\right)$ of exists, any optimal solution of $\left(P_{\leq_{\alpha}}\right)$ is an optimal solution of $\left(P^{\alpha}\right)$.

2. If an optimal solution of $(P)$ exists, any optimal solution of $\left(P_{\leq_{1}}\right)$ is an optimal solution of $(P)$.

3. Let $\left\{\alpha_{n}\right\}$ be a strictly increasing nonnegative sequence converging to 1 . Let $f(q)$ and $\mu_{g}(q)$ be continuous functions of $q$. Assume that an optimal solution $\tilde{q}$ of $\left(P^{1}\right)$ exists and an optimal solution $\tilde{q}_{n}$ of $\left(P_{\leq_{\alpha_{n}}}\right)$ exists for any $\alpha_{n}$. Then, every accumulation point of the sequence $\left\{\tilde{q}_{n}\right\}$ is an optimal solution of $\left(P^{1}\right)$.

To transform a simplex method into a constrained one, we simply replace every $f\left(q_{1}\right) \leq f\left(q_{2}\right)$ with $\left(f\left(q_{1}\right)\right.$, $\left.\mu_{g}\left(q_{1}\right)\right) \leq_{\alpha}\left(f\left(q_{2}\right), \mu_{g}\left(q_{2}\right)\right)$. The strict inequality is similarly changed. The value of $\alpha$ is normally kept at 1 but in some situations (e.g., when the feasible region is small), $\alpha(0)$ is chosen less than 1 and its value is increased to 1 by a control law such as $\alpha(t)=(1-\beta) \alpha(t-1)+\beta$, where $0<\beta<1$ (see Takahama and Sakai 2005).

Golden rule and weighted average: In Nazareth and Tseng (2002), it is argued that the values usually chosen for the coefficients are heuristic and instead propose to set $\delta_{r}=$ $\rho^{\log _{\rho}(2)}, \delta_{2}=\rho^{2}, \delta_{1}=\rho, \delta_{-1}=\rho^{-1}$, and $\xi=\rho^{2}$, where $\rho=(\sqrt{5}+1) / 2$. These selections are derived from a reinterpretation of univariate golden-section direct search. They prove that with these choices, in the one dimensional case, if $f$ is strictly unimodal on $\Re$, then, either $\left\{\operatorname{diam}_{o}\left(S^{(k)}\right)\right\} \rightarrow \infty$ and $f$ has no minimizer, or $\left\{\operatorname{diam}_{o}\left(S^{(k)}\right)\right\} \rightarrow 0$ linearly and $\left\{q^{(k)}\right\}$ converges to the unique minimizer of $f$.

Moreover, the centroid and the average value of the simplex are computed as weighted averages by choosing $\theta_{1} \in$ $(0,1)$ and setting

$\bar{q}^{(k)}=\mu_{2} q_{2}^{(k)}+\mu_{1} q_{1}^{(k)}$,

$\overline{f\left(q^{(k)}\right)}=\mu_{2} f\left(q_{2}^{(k)}\right)+\mu_{1} f\left(q_{1}^{(k)}\right)$,

where

$\mu_{i}=\left(1-\theta_{1}\right) \frac{f\left(q_{3}^{(k)}\right)-f\left(q_{i}^{(k)}\right)}{\sum_{j \in\{1,2\}}\left(f\left(q_{3}^{(k)}\right)-f\left(q_{j}^{(k)}\right)\right)}+\frac{\theta_{1}}{2}$,

$i \in\{1,2\}$, provided $f\left(q_{1}^{(k)}\right) \neq f\left(q_{3}^{(k)}\right) . \theta_{1}=1$ then corresponds to $\mu_{i}=1 / 2$.

Fortified descent and degeneracy safeguards: In Nazareth and Tseng (2002), a convergent version of the basic algorithm is proposed. To do this, they propose certain safeguards. They employ fortified descent which is stronger than strict descent and maintain the interior angles of the simplex $S^{(k)}$ bounded away from zero (which is equivalent to bounding $v\left(S^{k}\right)$ away from zero). In their modified algorithm, a $\theta_{2} \in\left(0, v\left(S^{(k)}\right)\right]$ and any continuous functions $\sigma: \mathfrak{R}^{+} \rightarrow \mathfrak{R}^{+}$and $\beta: \mathfrak{R}^{+} \rightarrow \mathfrak{R}^{+}$are chosen with 
the property that $\lim _{t \rightarrow 0}(\sigma(t) / t)=\lim _{t \rightarrow 0}(\beta(t)) / t=0$ and $\inf _{t \geq a} \sigma(t)>0$ for all $a>0$. In their modified algorithm, the inequalities in steps $2-5$ are replaced with those that involve the term $\sigma\left(\operatorname{diam}_{o}\left(S^{(k)}\right)\right)$. Also, the condition $v\left(S_{\delta_{2}}^{(k)}\right) \geq \theta_{2}$ appears in steps 3-5. They then prove that if $f$ is continuously differentiable and quasi-convex on $\mathfrak{R}^{2}$, is uniformly continuous on the level set $\Gamma_{\min }{ }_{q \in S^{(0)}} f(q)(f)$, and if $\inf _{q \in \mathfrak{R}^{2}} f(q)>-\infty$, then $\left\{\operatorname{diam}_{o}\left(S^{(k)}\right)\right\} \rightarrow 0$, and every cluster point of $\left\{q^{(k)}\right\}$ is a stationary point of $f$, where $\left\{S^{(k)}, q^{(k)}\right\}$ is the sequence generated by the modified algorithm.

Note that $\theta_{1}, \sigma(t)$ and $\beta(t)$ can be selected in such a way that the fortified descent is only slightly stronger than strict descent. From a practical point of view, there is not much difference between the two. Fortified descent is only used to assure convergence. The second modification, though, can be very crucial for avoiding degenerate simplexes. We will take up this idea later on to derive a constrained version of the algorithm.

Globalization: The simplex method is a local optimization strategy. As such, it quickly converges to a local minimum. A number of strategies have been proposed to make this algorithm a global search method. One promising method has been proposed in Luersen et al. (2004). The basic idea is to restart the search from a new initial position when a local search finishes. In our case, a local search is considered finished if it has reached a flat area or it cannot get smaller for more fine tuning. A list of past initial points $\Phi$ is maintained. Let $q_{I}^{(i)}, i=0, \ldots, M-1$ represent the initial points from which the simplex has already done a local search. The probability of selecting $q_{I}^{(i)}$ again for restart can be modelled by the Gaussian

$p_{i}(q)=\frac{1}{2 \pi \sigma^{2}} e^{-\frac{1}{\sigma^{2}}\left\|q-q_{I}^{(i)}\right\|^{2}}$.

The probability of sampling a past initial point is then given by

$p(q, \Phi)=\frac{1}{M} \sum_{i=0}^{M-1} p_{i}(q)$.

The normalized probability is

$\tilde{p}(q, \Phi)=\frac{p(q)}{\int_{\Omega} p(q) d \Omega}$.

We want the probability of sampling an already used initial point to approach zero and thus the probability density of sampling a new initial position can be given by

$\phi(q, \Phi)=\frac{H-\tilde{p}(q)}{\int_{\Omega}(H-\tilde{p}(q)) d \Omega}$,

where $H=\max _{q \in \Omega} \tilde{p}(q, \Phi)$. In practice, we can select the new position by the formula $q_{I}^{(M)}=\operatorname{argmin}_{q \in \Omega} p(q, \Phi)$.

\section{Shape-constrained version}

As is apparent from the foregoing discussions, the NelderMead can sometimes get very elongated in one direction, making the simplex degenerate. This is a key to its failure in many cases. Moreover, when applying an algorithm such as the Nelder-Mead simplex method to robotic formations, one always has to provide for safeguards against violation of important geometric constraints, most notably the ones imposed on minimum and maximum distances between vehicles participating in the task. The algorithm, as stated above, clearly has the potential to violate the angle constraints and it always violates the distance constraint, if it is going to converge at all. In this section, we present a variant which takes care of these constraints. Except for the safeguard for nondegeneracy, no constraint is normally put on distances between nodes for the simple reason that doing this will prevent the simplex from converging (upon successful convergence, the diameter of the simplex tends to zero). In our application, this does not present a problem. If the simplex gets smaller than some bound, it means that either we are near a local minimizer or else the simplex is going to stagnate. Localizing exactly a minimizer is not needed in the real world application that we are addressing here. Our main goal, by considering the Nelder-Mead method, is to get as close as possible to a certain feature (source or isocline) as fast (and as aggressively) as possible. The idea here is to check if a new point is within desirable bounds and if not project it unto the bound (Fig. 4). The bounds we are interested in are

$D_{l} \leq \operatorname{diam}_{i}\left(S^{(k)}\right)$,

$\operatorname{diam}_{o}\left(S^{(k)}\right) \leq D_{u}$,

$v\left(S^{(k)}\right) \leq v_{u}$.

Assume that $S^{(k)}$ satisfies these bounds. Instead of the original $\delta \in\left\{\delta_{r}, \delta_{2}, \delta_{1}, \delta_{-1}\right\}$, we apply a constrained value $\chi\left(\delta_{i}\right)$. We have $\delta_{r}=\chi\left(\delta_{r}\right)$, as reflection does not change the shape of the simplex. For the expansion operation, we set

$$
\begin{aligned}
\chi\left(\delta_{2}\right)=\min & \left\{\operatorname{argmin}_{\delta}\left(v\left(S_{\delta_{2}}^{(k)}\right)-v_{u}\right),\right. \\
& \left.\operatorname{argmin}_{\delta}\left(\operatorname{diam}_{o}\left(S_{\delta_{2}}^{(k)}\right)-v_{u}\right), \delta_{2}\right\} .
\end{aligned}
$$

For the outside and inside contractions, we set, respectively,

$$
\begin{aligned}
\chi\left(\delta_{1}\right)=\max & \left\{\operatorname{argmax}_{\delta}\left(v\left(S_{\delta_{1}}^{(k)}\right)-v_{u}\right),\right. \\
& \left.\operatorname{argmax}_{\delta}\left(\operatorname{diam}_{o}\left(S_{\delta_{1}}^{(k)}\right)-v_{u}\right), \delta_{1}\right\},
\end{aligned}
$$

and

$$
\begin{aligned}
\chi\left(\delta_{-1}\right)=\min & \left\{\operatorname{argmin}_{\delta}\left(v\left(S_{\delta_{-1}}^{(k)}\right)-v_{u}\right),\right. \\
& \left.\operatorname{argmin}_{\delta}\left(\operatorname{diam}_{o}\left(S_{\delta_{-1}}^{(k)}\right)-v_{u}\right), \delta_{-1}\right\} .
\end{aligned}
$$


Fig. 4 Constraining generated points to lie within the desired bounds. The computed points $z^{(k)}(\delta)$ are checked and, if necessary, are modified to $z^{(k)}(\chi(\delta))$. Likewise, $\beta_{l}$ is modified to $\chi\left(\beta_{l}\right)$

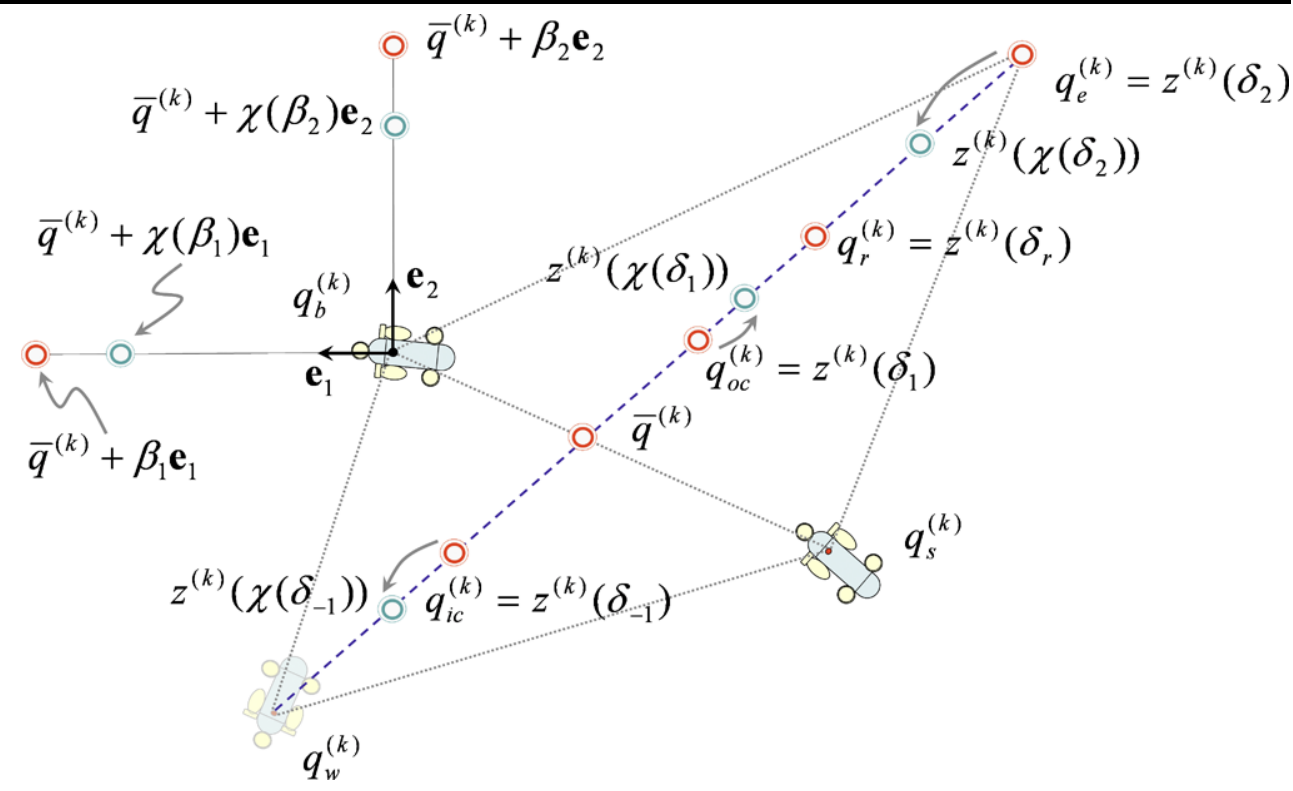

It is obvious that $\delta_{r} \leq \chi\left(\delta_{2}\right) \leq \delta_{2}, \delta_{1} \leq \chi\left(\delta_{1}\right) \leq \delta_{r}$, and $0 \leq \chi\left(\delta_{-1}\right) \leq \delta_{-1}$. Although the above optimization problems can be solved by simple line search, we prefer to make things simpler by replacing the above constraints with equivalent but more manageable and straightforward ones. For any simplex, we require that, for every $q_{i}$ and $q_{j}, i \neq j$, $i, j \in\{1,2,3\}$, we have $D_{l} \leq\left\|q_{i}-q_{j}\right\| \leq D_{u}$, and that, for every two edges $e_{i j}$ and $e_{i k}$, attached to $q_{i}$, we have $\varphi_{l} \leq \varphi\left(q_{i}, q_{j}, q_{k}\right) \leq \varphi_{u}$, where

$\varphi\left(q_{i}, q_{j}, q_{k}\right)=\operatorname{acos}\left(\frac{\left\langle\left(q_{j}-q_{i}\right),\left(q_{k}-q_{i}\right)\right\rangle}{\left\|q_{i}-q_{j}\right\|\left\|q_{i}-q_{k}\right\|}\right)$.

We denote the distance constraints by $C_{D_{l}}\left(q_{i}, q_{j}\right)$ and $C_{D_{u}}\left(q_{i}, q_{j}\right)$. The angle constraints are denoted by $C_{\varphi_{l}}\left(q_{i}\right.$, $\left.q_{j}, q_{k}\right)$ and $C_{\varphi_{u}}\left(q_{i}, q_{j}, q_{k}\right)$. Not all the constraints can be violated by all of the operations. Reflection, as was mentioned, does not violate any constraints. Expansion can only violate the maximum distance constraints $C_{D_{u}}\left(q_{1}^{(k)}, z^{(k)}\left(\delta_{2}\right)\right)$, and $C_{D_{u}}\left(q_{2}^{(k)}, z^{(k)}\left(\delta_{2}\right)\right)$, the minimum angle constraint $C_{\varphi_{l}}\left(z^{(k)}\left(\delta_{2}\right), q_{1}^{(k)}, q_{2}^{(k)}\right)$, and the maximum angle constraints $C_{\varphi_{u}}\left(q_{1}^{(k)}, q_{2}^{(k)}, z^{(k)}\left(\delta_{2}\right)\right)$, and $C_{\varphi_{u}}\left(q_{2}^{(k)}, q_{1}^{(k)}, z^{(k)}\left(\delta_{2}\right)\right)$.

Outside contraction can potentially violate the minimum distance constraints $C_{D_{l}}\left(q_{1}^{(k)}, z^{(k)}\left(\delta_{1}\right)\right)$, and $C_{D_{l}}\left(q_{2}^{(k)}\right.$, $\left.z^{(k)}\left(\delta_{1}\right)\right)$, the minimum angle constraints $C_{\varphi_{l}}\left(q_{1}^{(k)}, q_{2}^{(k)}\right.$, $\left.z^{(k)}\left(\delta_{1}\right)\right)$, and $C_{\varphi_{l}}\left(q_{2}^{(k)}, q_{1}^{(k)}, z^{(k)}\left(\delta_{1}\right)\right)$, and the maximum angle constraint $C_{\varphi_{u}}\left(z^{(k)}\left(\delta_{1}\right), q_{1}^{(k)}, q_{2}^{(k)}\right)$.

Likewise, inside contraction can violate the same constraints as outside contraction. We only consider and check for a subset of these constraints. For expansion, we consider $C_{D_{u}}\left(q_{i}^{(k)}, z^{(k)}\left(\delta_{2}\right)\right), i \in\{1,2\}$, and $C_{\varphi_{l}}\left(z^{(k)}\left(\delta_{2}\right), q_{1}^{(k)}, q_{2}^{(k)}\right)$. For outside and inside contractions, we check for $C_{D_{l}}\left(q_{i}^{(k)}\right.$, $\left.z^{(k)}\left(\delta_{j}\right)\right), j \in\{1,-1\}$, and $C_{\varphi_{l}}\left(q_{i}^{(k)}, q_{k}^{(k)}, z^{(k)}\left(\delta_{j}\right)\right), k \in$ $\{1,2\}, k \neq i$.

From the definition of $z^{(k)}(\delta)$, we have

$z^{(k)}(\delta)-\bar{q}^{(k)}=\delta\left(\bar{q}^{(k)}-q_{w}^{(k)}\right)$,

or

$$
\begin{array}{r}
\left\|z^{(k)}(\delta)-\bar{q}^{(k)}\right\| \frac{z^{(k)}(\delta)-\bar{q}^{(k)}}{\left\|z^{(k)}(\delta)-\bar{q}^{(k)}\right\|} \\
=\delta\left\|\bar{q}^{(k)}-q_{w}^{(k)}\right\| \frac{\bar{q}^{(k)}-q_{w}^{(k)}}{\left\|\bar{q}^{(k)}-q_{w}^{(k)}\right\|}
\end{array}
$$

which implies that

$\left\|z^{(k)}(\delta)-\bar{q}^{(k)}\right\|=\delta\left\|\bar{q}^{(k)}-q_{w}^{(k)}\right\|$.

Letting $\Delta^{(k)}(\delta)=\left\|z^{(k)}(\delta)-\bar{q}^{(k)}\right\|$, we have

$\delta=\frac{\Delta^{(k)}(\delta)}{\left\|\bar{q}^{(k)}-q_{w}^{(k)}\right\|}$.

For the distance constraint we have

$$
\begin{aligned}
D^{2}= & \left\|q_{i}^{(k)}-\bar{q}^{(k)}\right\|^{2}+\Delta_{i}^{(k)}(D)^{2} \\
& -2 \Delta_{i}^{(k)}(D)\left\|q_{i}^{(k)}-\bar{q}^{(k)}\right\| \cos \left(\varphi\left(\bar{q}^{(k)}, q_{i}^{(k)}, \zeta^{(k)}\right)\right),
\end{aligned}
$$

where $i \in\{1,2\}, \Delta_{i}^{(k)}(D)$ corresponds to a $\delta$ for which $\left\|z^{(k)}(\delta)-q_{i}^{(k)}\right\|=D$, and

$\zeta^{(k)}=\bar{q}^{(k)}+\mu \frac{\bar{q}^{(k)}-q_{w}^{(k)}}{\left\|\bar{q}^{(k)}-q_{w}^{(k)}\right\|}$. 
Fig. 5 Calculations for the distance constraint. (a) Upper bound for the distance when expansion is being attempted. (b) Lower bound for distance when contracting outside or inside (a) $z^{(k)}\left(\delta_{1}^{(k)}\left(D_{u}\right)\right)$

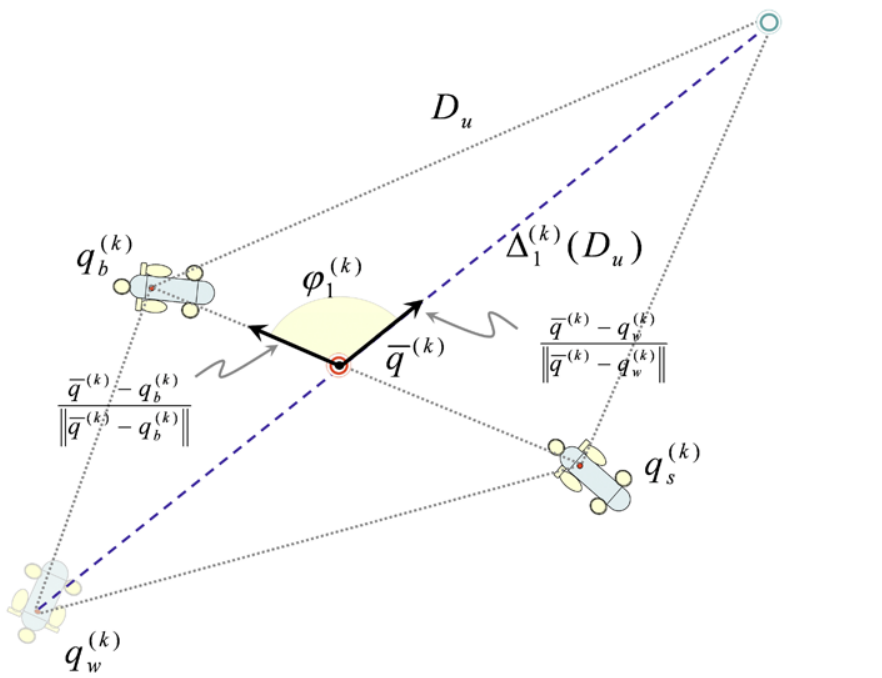

(b)

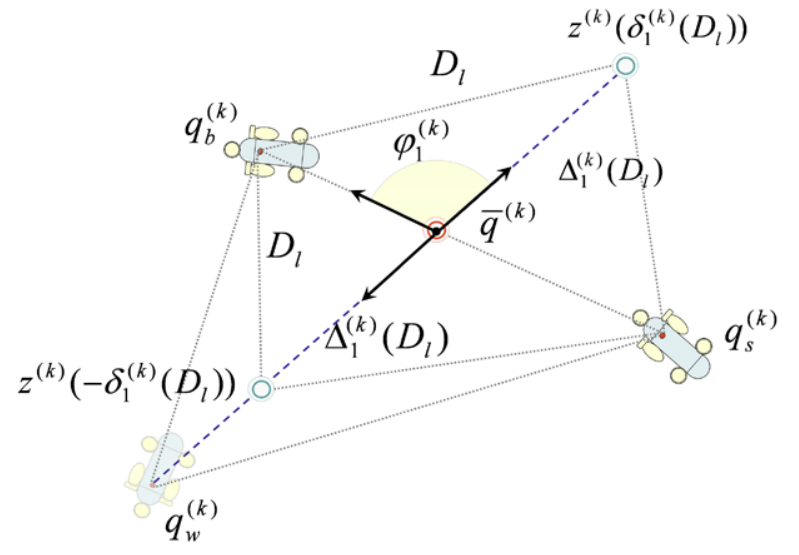

$\mu$ is equal to $\operatorname{sign}(\delta)$. Of course, we are trying to compute $\delta$, so we put $\mu=1$ for expansion and outside contraction and we put $\mu=-1$ for inside contraction. Solving for $\Delta_{i}^{(k)}(D)$, we get

$$
\begin{aligned}
\Delta_{i}^{(k)}(D)= & \left\|q_{i}^{(k)}-\bar{q}^{(k)}\right\| \cos \left(\varphi_{i}^{(k)}\right) \\
& +\left(\left\|q_{i}^{(k)}-\bar{q}^{(k)}\right\|^{2}\left(\cos \left(\varphi_{i}^{(k)}\right)^{2}-1\right)+D^{2}\right)^{1 / 2}
\end{aligned}
$$

where $\varphi_{i}^{(k)}=\varphi\left(\bar{q}^{(k)}, q_{i}^{(k)}, \zeta^{(k)}\right)$. Here $D \in\left\{D_{l}, D_{u}\right\}$. Now,

$\delta_{i}^{(k)}(D)=\frac{\Delta_{i}^{(k)}(D)}{\left\|\bar{q}^{(k)}-q_{w}^{(k)}\right\|}$

is the expression for the $\delta$ which places the new vertex on the boundary of the distance constraint. See Fig. 5 for an illustration of the introduced variables. If the real solution of the second order equation does not exist, the simplex is already outside the safe region.
For the minimum angle constraint $C_{\varphi_{l}}\left(z^{(k)}(\delta), q_{1}^{(k)}, q_{2}^{(k)}\right)$ (Fig. 6), we note that, by the sine law,

$\Delta_{z}^{(k)}\left(\varphi_{l}\right)=\left\|\bar{q}^{(k)}-q_{1}^{(k)}\right\| \frac{\sin \left(\tilde{\gamma}_{1}^{(k)}\right)}{\sin \left(\tilde{\varphi}_{l}\right)}$,

where

$\tilde{\varphi}_{l}=\frac{\left\|\bar{q}^{(k)}-q_{1}^{(k)}\right\|}{\left\|q_{2}^{(k)}-q_{1}^{(k)}\right\|} \varphi_{l}$,

$\tilde{\gamma}_{1}^{(k)}=\pi-\left(\varphi_{1}^{(k)}+\tilde{\varphi}_{l}\right)$.

The vertex $q_{2}^{(k)}$ could have alternatively been used. The corresponding $\delta$ is thus

$\delta_{z}^{(k)}\left(\varphi_{l}\right)=\Delta_{z}^{(k)}\left(\varphi_{l}\right) /\left\|\bar{q}^{(k)}-q_{w}^{(l)}\right\|$.

See Fig. 6(a). 
Fig. 6 Calculations for the angle constraint. (a) Lower bound for the angle when expansion is being attempted. (b) Lower bound for angle when contracting outside or inside

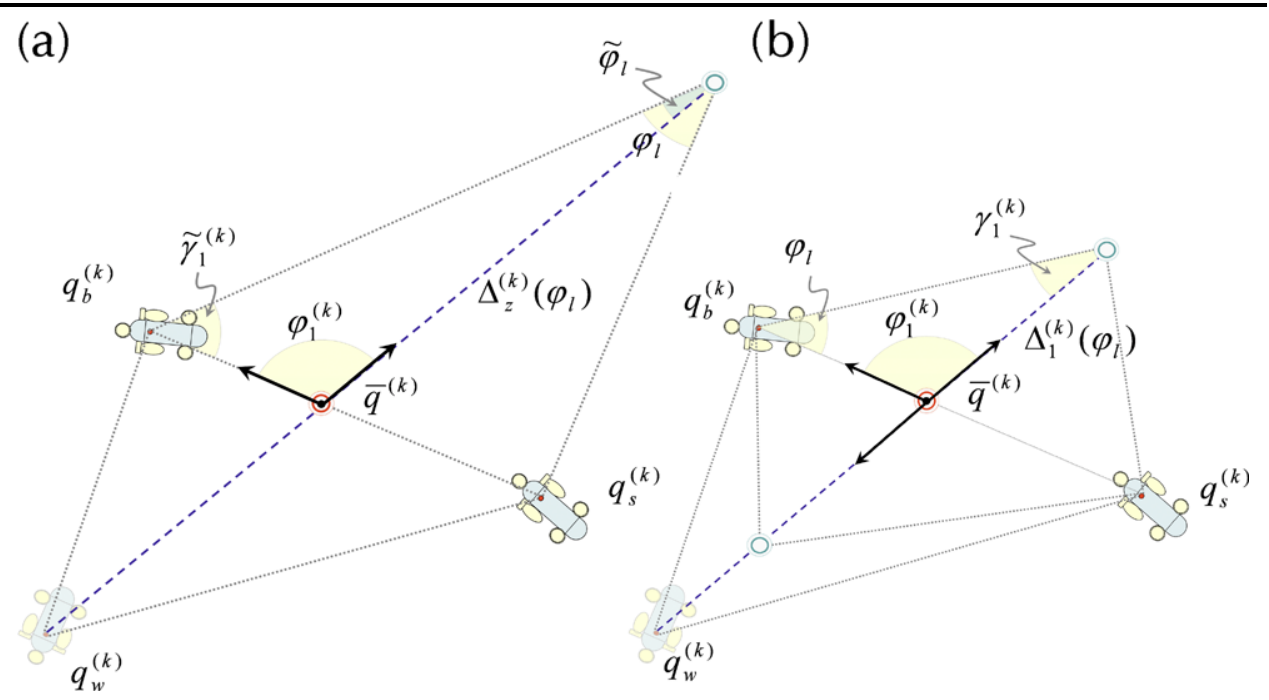

For the constraint $C_{\varphi_{l}}\left(q_{i}^{(k)}, q_{k}^{(k)}, z^{(k)}\left(\delta_{j}\right)\right)$ imposed on outside and inside contraction operations (Fig. 6(b)), we use the formula

$\Delta_{i}^{(k)}\left(\varphi_{l}\right)=\left\|\bar{q}^{(k)}-q_{i}^{(k)}\right\| \frac{\sin \left(\varphi_{l}\right)}{\sin \left(\gamma_{i}^{(k)}\right)}$,

where $\gamma_{i}^{(k)}=\pi-\left(\varphi_{i}^{(k)}+\varphi_{l}\right)$, and so

$\delta_{i}^{(k)}\left(\varphi_{l}\right)=\mu \Delta_{i}^{(k)}\left(\varphi_{l}\right) /\left\|\bar{q}^{(k)}-q_{w}^{(k)}\right\|$.

The following lemma sums up the main strategy.

Lemma 4 Define the simplex shape space $\Gamma \subseteq \mathfrak{R}^{3} \otimes \mathfrak{R}^{3}$ as the set of all the consistent tuples

$\left(\left\|q_{1}-q_{2}\right\|,\left\|q_{1}-q_{3}\right\|,\left\|q_{2}-q_{3}\right\|, \varphi\left(q_{1}, q_{2}, q_{3}\right)\right.$,

$\left.\varphi\left(q_{2}, q_{1}, q_{3}\right), \varphi\left(q_{3}, q_{1}, q_{2}\right)\right)$,

denoted $\widehat{S}$. Let

$\Pi=\prod_{i=1}^{3}\left[D_{l}, D_{u}\right] \otimes \prod_{i=1}^{3}\left[\varphi_{l}, \varphi_{u}\right]$

be the constrain hypercube in $\Gamma$. Assume that $\widehat{S}^{(0)} \in \Pi$. If, instead of $\delta_{2}, \delta_{1}, \delta_{-1}$, and $\beta_{l}$ in the original algorithm, we use, respectively,

$$
\begin{gathered}
\chi\left(\delta_{2}\right)=\min \left\{\delta_{1}^{(k)}\left(D_{u}\right), \delta_{2}^{(k)}\left(D_{u}\right),\right. \\
\left.\delta_{z}^{(k)}\left(\varphi_{l}\right), \delta_{1}^{(k)}\left(\varphi_{u}\right), \delta_{2}^{(k)}\left(\varphi_{u}\right), \delta_{2}\right\}, \\
\chi\left(\delta_{1}\right)=\max \left\{\delta_{1}^{(k)}\left(D_{l}\right), \delta_{2}^{(k)}\left(D_{l}\right),\right. \\
\left.\delta_{1}^{(k)}\left(\varphi_{l}\right), \delta_{2}^{(k)}\left(\varphi_{l}\right), \delta_{z}^{(k)}\left(\varphi_{u}\right), \delta_{1}\right\},
\end{gathered}
$$

$$
\begin{gathered}
\chi\left(\delta_{-1}\right)=\min \left\{-\delta_{1}^{(k)}\left(D_{l}\right),-\delta_{2}^{(k)}\left(D_{l}\right),-\delta_{1}^{(k)}\left(\varphi_{l}\right),\right. \\
\left.-\delta_{2}^{(k)}\left(\varphi_{l}\right),-\delta_{z}^{(k)}\left(\varphi_{u}\right), \delta_{-1}\right\}, \\
\chi\left(\beta_{l}\right)=\min \left\{(1 / 2) \sigma_{-}\left(S^{(k)}\right), D_{l}\right\}, \\
\eta\left(\operatorname{sign}\left(\left\langle D\left(f: S^{(k)}\right), e_{l}\right\rangle\right)\right),
\end{gathered}
$$

then we will have $\widehat{S}^{(k)} \in \Pi$, for every $k \geq 0$.

\section{The modified algorithm}

We can now state the new algorithm. First, let us introduce the special notation we will use throughout the algorithm. We use $\tau_{\mu}$ to denote the mode of operation. If $\tau_{\mu}=1$, the task is that of minimization and if $\tau_{\mu}=2$, we are maximizing. Let $u_{1}, u_{2} \in \mathfrak{R}^{2}$.

1. We use the notation $f\left(u_{1}\right)\left[\tau_{\mu},<\right] f\left(u_{2}\right)$ (or, equivalently $\left.f\left(u_{2}\right)\left[\tau_{\mu},>\right] f\left(u_{1}\right)\right)$ to denote the fact that $f\left(u_{1}\right)$ is strictly better than $f\left(u_{2}\right)$. For minimization, this is equivalent to $f\left(u_{1}\right)<f\left(u_{2}\right)$, while for maximization, it is equal to $f\left(u_{1}\right)>f\left(u_{2}\right)$.

2. We use the notation $f\left(u_{1}\right)\left[\tau_{\mu}, \leq\right] f\left(u_{2}\right)$ (or, equivalently $\left.f\left(u_{2}\right)\left[\tau_{\mu}, \geq\right] f\left(u_{1}\right)\right)$ to denote the fact that $f\left(u_{1}\right)$ is better than or equal to $f\left(u_{2}\right)$.

3. We use the notations $\left(f\left(u_{1}\right), \mu_{g}\left(u_{1}\right)\right)\left[\tau_{\mu}, \leq_{\alpha}\right]\left(f\left(u_{2}\right)\right.$, $\left.\mu_{g}\left(u_{2}\right)\right), \quad\left(f\left(u_{1}\right), \quad \mu_{g}\left(u_{1}\right)\right)\left[\tau_{\mu},<_{\alpha}\right]\left(f\left(u_{2}\right), \quad \mu_{g}\left(u_{2}\right)\right)$, $\left(f\left(u_{1}\right), \mu_{g}\left(u_{1}\right)\right)\left[\tau_{\mu}, \geq_{\alpha}\right]\left(f\left(u_{2}\right), \mu_{g}\left(u_{2}\right)\right)$, and $\left(f\left(u_{1}\right)\right.$, $\left.\mu_{g}\left(u_{1}\right)\right)\left[\tau_{\mu},>_{\alpha}\right]\left(f\left(u_{2}\right), \mu_{g}\left(u_{2}\right)\right)$ to convey the fact that $\alpha$-level comparisons have been used.

$n_{\text {or }}$ denotes the number of oriented restarts in a row. Following Conn et al. (1997), we terminate the search after three such restarts. A denotes the initial size of the simplex. The algorithm is composed of two loops. The outer loop generates random initial positions and the inner one iterates the 
local search until termination. After a sufficient number of probabilistic restarts, the algorithm terminates.

Algorithm 2 Shape-Constrained Nelder-Mead $\left(q_{I}^{(0)}, f\right.$, $\varepsilon, A)$.

Set $l=0, \Phi=\{\}$, and go to step 2-1.

Step 2-1 (Generation of initial point)

Set $n_{o r}=0$. If $l \neq 0, \Phi=\Phi \cup\left\{q_{I}^{(l-1)}\right\}$. Set $q_{I}^{(l)}$ to a sample point drawn from the probability density $\phi(q, \Phi)$. Go to step 2-2.

\section{Step 2-2 (Construction of initial simplex)}

Construct the initial simplex by setting (see Haftka and Guerdal 1993)

$$
\begin{array}{r}
q_{1}^{(0)}=q_{I}^{(l)}, \\
q_{2}^{(0)}=q_{1}^{(0)}+\frac{A}{2 \sqrt{2}}(\sqrt{3}+1) e_{1}+\frac{A}{2 \sqrt{2}}(\sqrt{3}+1) e_{2} \\
q_{3}^{(0)}=q_{1}^{(0)}+\frac{A}{2 \sqrt{2}}(\sqrt{3}+1) e_{1}+\frac{A}{2 \sqrt{2}}(\sqrt{3}+1) e_{2},
\end{array}
$$

set $k=0$ and go to step 2-3.

\section{Step 2-3 (Checking for termination)}

The current local search should be terminated if (see Nazareth and Tseng 2002)

$$
\left\|\left[\begin{array}{c}
\left(q_{2}^{(k)}-q_{1}^{(k)}\right)^{T} \\
\left(q_{3}^{(k)}-q_{1}^{(k)}\right)^{T}
\end{array}\right]^{-1}\left[\begin{array}{l}
f\left(q_{2}^{(k)}\right)-f\left(q_{1}^{(k)}\right) \\
f\left(q_{3}^{(k)}\right)-f\left(q_{1}^{(k)}\right)
\end{array}\right]\right\| \leq \varepsilon .
$$

If is continuously differentiable, $v\left(S^{(k)}\right)$ is bounded away from zero and $\operatorname{diam}\left(S^{(k)}\right)$ tends to zero, then the above criterion means that $\nabla f\left(q_{1}^{(k)}\right) \approx 0$. If this is true, set $l \leftarrow l+1$ and go to step 2-1.

Step 2-4 (Oriented restart)

If $k=0$, go to step $2-5$. If

$$
\overline{\left(f\left(q^{(k)}\right)\right.}-\overline{f\left(q^{(k-1)}\right)}\left[\tau_{\mu},<\right] 0
$$

but

$$
\begin{aligned}
& \left.\overline{\left(f\left(q^{(k)}\right)\right.}-\overline{f\left(q^{(k-1)}\right)}\right) \\
& \quad\left[\tau_{\mu}, \geq\right]-\alpha_{0} \frac{\sigma_{+}\left(S^{(0)}\right)}{\left\|D\left(f: S^{(0)}\right)\right\|}\left\|D\left(f: S^{(k-1)}\right)\right\|^{2}
\end{aligned}
$$

then, if $n_{o r}=2$, set $l \leftarrow l+1$ and go to step 2-1. Otherwise, set $n_{o r} \leftarrow n_{o r}+1$, and

$\tilde{S}=S\left[q_{1}^{(k)}, q_{1}^{(k)}+\Psi\left(e_{1}\right) e_{1}, q_{1}^{(k)}+\Psi\left(e_{2}\right) e_{2}\right]$

where

$\Psi(u)=\frac{1}{2} \sigma_{-}\left(S^{(k)}\right) \eta\left(\operatorname{sign}\left(\left\langle D\left(f: S^{(k)}\right), u\right\rangle\right)\right)$.

Set $S^{(k)}=\tilde{S}$.
Step 2-5 (Sort the vertices)

Sort and label the vertices according to functional value as $q_{w}^{(k)}, q_{s}^{(k)}$, and $q_{b}^{(k)}$ such that

$$
\begin{aligned}
\left(f\left(q_{b}^{(k)}\right), \mu_{g}\left(q_{b}^{(k)}\right)\right)\left[\tau_{\mu},\right. & \leq \alpha(t)]\left(f\left(q_{s}^{(k)}\right), \mu_{g}\left(q_{s}^{(k)}\right)\right) \\
{\left[\tau_{\mu},\right.} & \leq \alpha(t)]\left(f\left(q_{w}^{(k)}\right), \mu_{g}\left(q_{w}^{(k)}\right)\right) .
\end{aligned}
$$

\section{Step 2-6 (Reflection)}

If

$$
\left(f\left(q_{b}^{(k)}\right), \mu_{g}\left(q_{b}^{(k)}\right)\right)\left[\tau_{\mu}, \leq_{\alpha(t)}\right]\left(f\left(z^{(k)}\left(\delta_{r}\right)\right), \mu_{g}\left(z^{(k)}\left(\delta_{r}\right)\right)\right)
$$

and

$$
\left(f\left(z^{(k)}\left(\delta_{r}\right)\right), \mu_{g}\left(z^{(k)}\left(\delta_{r}\right)\right)\right)\left[\tau_{\mu},<_{\alpha(t)}\right]\left(f\left(q_{s}^{(k)}\right), \mu_{g}\left(q_{s}^{(k)}\right)\right),
$$

set $S^{(k+1)}=S_{\delta_{r}}^{(k)}$ and go to step 2-5.

\section{Step 2-7 (Expansion)}

Let

$$
\begin{gathered}
B:\left(f\left(z^{(k)}\left(\delta_{r}\right)\right), \mu_{g}\left(z^{(k)}\left(\delta_{r}\right)\right)\right) \\
{\left[\tau_{\mu},<_{\alpha(t)}\right]\left(f\left(q_{b}^{(k)}\right), \mu_{g}\left(q_{b}^{(k)}\right)\right) .}
\end{gathered}
$$

If $\bar{B}$, go to step $2-8$.

If $B$ and

$$
\begin{aligned}
& \left(f\left(z^{(k)}\left(\chi\left(\delta_{2}\right)\right)\right), \mu_{g}\left(z^{(k)}\left(\chi\left(\delta_{2}\right)\right)\right)\right) \\
& {\left[\tau_{\mu},<_{\alpha(t)}\right]\left(f\left(z^{(k)}\left(\delta_{r}\right)\right), \mu_{g}\left(z^{(k)}\left(\delta_{r}\right)\right)\right),}
\end{aligned}
$$

set $S^{(k+1)}=S_{\chi\left(\delta_{2}\right)}^{(k)}$. Otherwise, set $S^{(k+1)}=S_{\delta_{r}}^{(k)}$. Go to step 2-5.

\section{Step 2-8 (Outside contraction)}

Let

$$
\begin{aligned}
& B_{1}:\left(f\left(q_{s}^{(k)}\right), \mu_{g}\left(q_{s}^{(k)}\right)\right) \\
& \quad\left[\tau_{\mu}, \leq_{\alpha(t)}\right]\left(f\left(z^{(k)}\left(\delta_{r}\right)\right), \mu_{g}\left(z^{(k)}\left(\delta_{r}\right)\right)\right),
\end{aligned}
$$

and

$$
\begin{gathered}
B:\left(f\left(z^{(k)}\left(\delta_{r}\right)\right), \mu_{g}\left(z^{(k)}\left(\delta_{r}\right)\right)\right) \\
{\left[\tau_{\mu},<_{\alpha(t)}\right]\left(f\left(q_{w}^{(k)}\right), \mu_{g}\left(q_{w}^{(k)}\right)\right) .}
\end{gathered}
$$

Let $B=B_{1} \wedge B_{2}$.

If $\bar{B}$, go to step 2-9.

If $B$ and

$$
\begin{gathered}
\left(f\left(z^{(k)}\left(\chi\left(\delta_{1}\right)\right)\right), \mu_{g}\left(z^{(k)}\left(\chi\left(\delta_{1}\right)\right)\right)\right) \\
{\left[\tau_{\mu},<_{\alpha(t)}\right]\left(f\left(q_{w}^{(k)}\right), \mu_{g}\left(q_{w}^{(k)}\right)\right),}
\end{gathered}
$$

set $S^{(k+1)}=S_{\chi\left(\delta_{1}\right)}^{(k)}$ and go to step 2-5. 
Otherwise, set $l \leftarrow l+1$ and go to step 2-1.

\section{Step 2-9 (Inside contraction)}

$$
\text { If }\left|\chi\left(\delta_{-1}\right)\right| \geq \varepsilon
$$

$$
\left(f\left(z^{(k)}\left(\delta_{r}\right)\right), \mu_{g}\left(z^{(k)}\left(\delta_{r}\right)\right)\right)\left[\tau_{\mu}, \geq_{\alpha(t)}\right]\left(f\left(q_{w}^{(k)}\right), \mu_{g}\left(q_{w}^{(k)}\right)\right)
$$

and

$$
\begin{gathered}
\left(f\left(z^{(k)}\left(\chi\left(\delta_{-1}\right)\right)\right), \mu_{g}\left(z^{(k)}\left(\chi\left(\delta_{-1}\right)\right)\right)\right) \\
{\left[\tau_{\mu},<_{\alpha(t)}\right]\left(f\left(q_{w}^{(k)}\right), \mu_{g}\left(q_{w}^{(k)}\right)\right),}
\end{gathered}
$$

set $S^{(k+1)}=S_{\chi\left(\delta_{-1}\right)}^{(k)}$ and go to step 2-5.

Otherwise, set $l \leftarrow l+1$ and go to step 2-1.

Considering this modified version, a number of explanations are in order:

1. We did not use fortified descent. As was mentioned previously, fortified descent is basically a means to ascertain convergence. Moreover, it can be designed to be very similar to strict descent. If the underlying function is reasonably well-behaving, both versions of the algorithm should behave exactly the same behaviour.

2. As is apparent from the convergence results, in cases where convergence is possible, the size of the simplex should go to zero. The shape-constrained version will stagnate when the size of the simplex can not be made smaller. In such situations, the simplex is very close (in the sense of the scale of the environment with respect to the minimum allowable size) to an optimal point. In practical applications, this is all we need.

3. In step $2-9$, we check to see if $\mid \chi\left(\delta_{-1}\right)$ is larger than some value. If this check is not done, the simplex may indefinitely try the reflection step and attempt inside contraction in case of failure only to end up in the same state as before. This indicates stagnation due to size limitations.

4. The convergence results should all hold true for this modified version. Firstly, the globalization strategy has no effect on the performance of local searches. Secondly, the $\alpha$-constrained comparisons do not change the properties of the algorithm. The only major change is the replacement of the constant coefficients with time-dependent ones. This, too, poses no difficulties since, for the algorithm equipped with oriented restarts, it is only required that the relations (3) hold true and we can choose $D_{l}, D_{u}$ and $\varphi_{l}$ in such a way that

$$
\begin{aligned}
-1 & <\chi\left(\delta_{-1}\right) \leq \delta_{-1}<0<\delta_{1} \leq \chi\left(\delta_{1}\right)<\delta_{r} \\
& <\chi\left(\delta_{2}\right) \leq \delta_{2} .
\end{aligned}
$$

5. No tests for degeneracy is done as the shape constraints make sure that the angles stay away from zero.

\section{Implementation by robotic formations}

In this section, we proceed to design a robotic system composed of three vehicles which implements the Nelder-Mead simplex method. The robots $\boldsymbol{R}_{1}, \boldsymbol{R}_{2}$ and $\boldsymbol{R}_{3}$ are assumed to be equipped with a compass module, flow meter, range and bearing sensors, as well as specific sensors for the application to measure the field value. It is assumed that the robots can communicate with each other using a fixed schedule. The time-dependent positions of the robots are denoted by $q_{1}(t), q_{2}(t)$, and $q_{3}(t)$. The formation itself is represented by the underlying simplex implicitly defined by the positions of the robots with respect to each other.

The function $f$ is now replaced with the environmental field $F: \mathfrak{R}^{2} \rightarrow \mathfrak{R}$. The robots measure a possibly noisy value for the field which is denoted $\tilde{F}(q(t))$. The field can be the bottom terrain, in which case altimeter sensors can be used. It can alternatively be a concentration. The field can be time-varying, denoted by $F(q, t)$ but it has to satisfy some conditions which will be stated later on.

The formation moves constrained to a plane parallel to the surface of the ocean. The vehicles are modelled as nonholonomic devices on this plane.

If the search region is constrained, sensors should be provided to measure $g(q(t))$. For the case of obstacles, optical or acoustic sensors are usually used. If the feasible region is defined by the operators, dead-reckoning might be sometimes used. If more accuracy is required, then resurfacing of the formation and using GPS is the only choice.

Likewise, when restarts are needed, the formation has to resurface. An alternative to resurfacing might be to employ a fourth vehicle especially designed for global localization purposes.

Assume that at the start of the mission (or the start of a local search), the robots form a nonsingular simplex $S^{(0)}$ and that all the distance and angle bounds are respected. Let $\tilde{F}\left(q_{1}\left(t_{0}\right)\right), \tilde{F}\left(q_{2}\left(t_{0}\right)\right)$ and $\tilde{F}\left(q_{3}\left(t_{0}\right)\right)$ be the measurements at time $t_{0}$. For the moment, let us assume that the robots can keep stationary perfectly such that $q_{i}\left(t_{0}+T\right)=q_{i}\left(t_{0}\right)$, for all $T \geq 0$. We can sort the values and relabel the robots, together with their positions (dropping the time dependence), as $\boldsymbol{R}_{w}, \boldsymbol{R}_{s}$ and $\boldsymbol{R}_{b}$ (correspondingly, $q_{w}^{(0)}, q_{s}^{(0)}$ and $q_{b}^{(0)}$ ), such that

$$
\begin{aligned}
\left(\tilde{F}\left(q_{b}^{(0)}\right), \mu_{g}\left(q_{b}^{(0)}\right)\right)\left[\tau_{\mu},\right. & \left.\leq_{\alpha}\right]\left(\tilde{F}\left(q_{s}^{(0)}\right), \mu_{g}\left(q_{s}^{(0)}\right)\right) \\
{\left[\tau_{\mu},\right.} & \left.\leq_{\alpha}\right]\left(\tilde{F}\left(q_{w}^{(0)}\right), \mu_{g}\left(q_{w}^{(0)}\right)\right) .
\end{aligned}
$$

To enact operations of the proposed algorithm, some of the robots have to do exploratory manoeuvres. In our system, we consider $\boldsymbol{R}_{b}$ as the pivot robot which is to keep station during an operation. Briefly, the system first tries reflection. To do this, $\boldsymbol{R}_{S}$ goes to $z^{(0)}\left(\delta_{r}\right)$ (while $\boldsymbol{R}_{w}$ is stationary) and measures the field value at that point. If the try is successful, $\boldsymbol{R}_{s}$ resumes the new location while $\boldsymbol{R}_{w}$ goes to $q_{s}^{(0)}$. 
If expansion is promising, $\boldsymbol{R}_{s}$ goes to $z^{(0)}\left(\chi\left(\delta_{2}\right)\right)$ instead. If successful, it remains there, otherwise, it returns back to $z^{(0)}\left(\delta_{r}\right)$. For outside contraction, $\boldsymbol{R}_{s}$ goes to $z^{(0)}\left(\chi\left(\delta_{1}\right)\right)$. For inside contraction, though, $\boldsymbol{R}_{s}$ returns back to $q_{s}^{(0)}$, while $\boldsymbol{R}_{w}$ goes to $z^{(0)}\left(\chi\left(\delta_{-1}\right)\right)$. If shrink is allowed, $\boldsymbol{R}_{s}$ goes to $r(s)$, where

$$
\begin{aligned}
r(u)= & q_{b}^{(0)}+\max \left\{\frac{1}{\xi}\left\|q_{u}^{(0)}-q_{b}^{(0)}\right\|, D_{l}\right\} \\
& \cdot \frac{\left(q_{s}^{(0)}-q_{b}^{(0)}\right)}{\left\|q_{u}^{(0)}-q_{b}^{(0)}\right\|},
\end{aligned}
$$

while $\boldsymbol{R}_{w}$ goes to $r(w)$. In the case of oriented restart, $\boldsymbol{R}_{s}$ goes to $q_{b}^{(0)}+\Psi\left(e_{1}\right) e_{1}$ or $q_{b}^{(0)}+\Psi\left(e_{2}\right) e_{2}$, whichever is closer to $q_{s}^{(0)}$, and $\boldsymbol{R}_{w}$ goes to the other point. This latter operation requires the use of the compass to determine directions of the global principal coordinates. These manoeuvres are depicted in Fig. 8.

Let us now consider a more realistic setting. At the beginning of the mission, the robot $\boldsymbol{R}_{1}$ (the choice is arbitrary) is assigned the role of the pivot robot. This means that the other vehicles should adjust their positions with respect to the pivot. The pivot itself should keep station at all times. This role may be given to other robots, as will be explained later. The positions of $\boldsymbol{R}_{2}$ and $\boldsymbol{R}_{3}$ with respect to $\boldsymbol{R}_{1}$ can be represented by the pairs $\left(D_{21}(t), \theta_{21}(t)\right)$ and $\left(D_{31}(t), \theta_{31}(t)\right)$, where $D_{i 1}(t)$ is the distance measured by the range sensor and $\theta_{i 1}(t)$ is he angle measured by the bearing sensor. Let us denote by $D_{i 1}^{d}$ and $\theta_{i 1}^{d}$, the desired distance and bearing. At the beginning of the mission, we put

$$
\begin{aligned}
& D_{i 1}^{d}=\left(\frac{2 A}{\sin (\pi / 3)}\right)^{1 / 2}, \\
& \theta_{21}^{d}=0, \text { and } \theta_{31}^{d}=\pi / 2 .
\end{aligned}
$$

Note that this is different from the initialization scheme in the algorithm but has the same effect. They try to keep station in this position by counteracting the flow of the medium until the first synchronization point $t_{0}$. At this time, field measurements are done and the robots are sorted. The role of the pivot is now relegated to $\boldsymbol{R}_{b}$. The relative measurements should now be calculated with respect to the new pivot. This is summarized in the following lemma (Fig. 7).

Lemma 5 Let $(b, s, w)=\partial(1,2,3)$ be any permutation of the ordered triple $(1,2,3)$. Then we have

$$
\begin{aligned}
& D_{s b}^{d}=\sqrt{\left(D_{\partial^{-1}(b) 1}^{d}\right)^{2}+\left(D_{\partial^{-1}(s) 1}^{d}\right)^{2}-2 B_{s}}, \\
& B_{s}=D_{\partial^{-1}(b) 1}^{d} D_{\partial^{-1}(s) 1}^{d} \cos \left(\varphi_{1,23}\right), \\
& D_{w b}^{d}=\sqrt{\left(D_{\partial^{-1}(b) 1}^{d}\right)^{2}+\left(D_{\partial^{-1}(w) 1}^{d}\right)^{2}-2 B_{w}},
\end{aligned}
$$

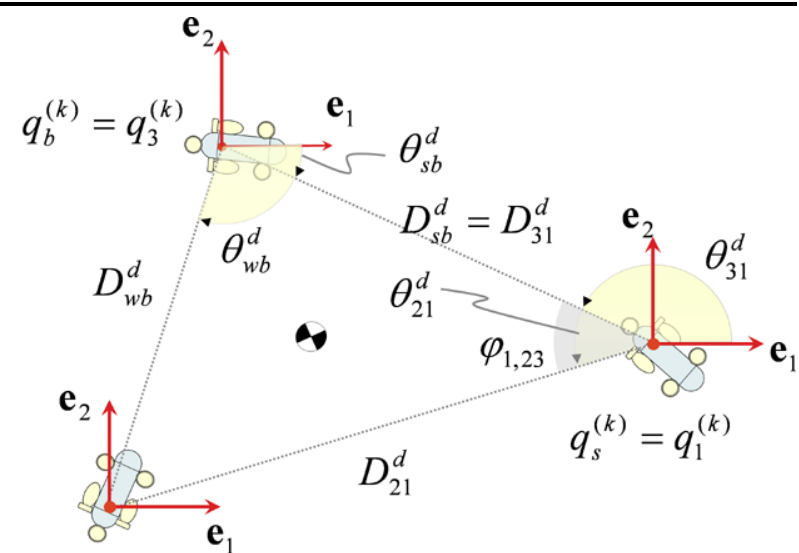

$q_{w}^{(k)}=q_{2}^{(k)}$

Fig. 7 Relative coordinates for the permutation $(3,1,2)=\partial(1,2,3)$

$B_{w}=D_{\partial^{-1}(b) 1}^{d} D_{\partial^{-1}(w) 1}^{d} \cos \left(\varphi_{1,23}\right)$,

$\theta_{s b}^{d}=\operatorname{acos}\left(\frac{v_{s b}}{\left\|v_{s b}\right\|}\right)$,

$\theta_{w b}^{d}=\operatorname{acos}\left(\frac{v_{w b}}{\left\|v_{w b}\right\|}\right)$,

where

$$
\begin{gathered}
\cos \left(\varphi_{1,23}\right)=\operatorname{acos}\left(\left(\begin{array}{c}
\cos \left(\theta_{21}^{d}\right) \\
\sin \left(\theta_{21}^{d}\right)
\end{array}\right) \cdot\left(\begin{array}{c}
\cos \left(\theta_{31}^{d}\right) \\
\sin \left(\theta_{31}^{d}\right)
\end{array}\right)\right), \\
v_{w b}=c\left(\partial^{-1}(b)\right) D_{\partial^{-1}(w) 1}^{d}\left(\begin{array}{c}
\cos \left(\theta_{\partial^{-1}(w) 1}^{d}\right) \\
\sin \left(\theta_{\partial^{-1}(w) 1}^{d}\right)
\end{array}\right) \\
+D_{\partial^{-1}(b) 1}^{d} \wedge d\left(\begin{array}{c}
\cos \left(\theta_{\partial^{-1}(b) 1}^{d}\right) \\
\sin \left(\theta_{\partial^{-1}(b) 1}^{d}\right)
\end{array}\right) \\
v_{s b=}\left(\partial^{-1}(b)\right) D_{\partial^{-1}(s) 1}^{d}\left(\begin{array}{c}
\cos \left(\theta_{\partial^{-1}(s) 1}^{d}\right) \\
\sin \left(\theta_{\partial^{-1}(s) 1}^{d}\right)
\end{array}\right) \\
+D_{\partial^{-1}(b) 1}^{d} \wedge d\left(\begin{array}{c}
\cos \left(\theta_{\partial^{-1}(b) 1}^{d}\right) \\
\sin \left(\theta_{\partial^{-1}(b) 1}^{d}\right)
\end{array}\right) .
\end{gathered}
$$

$c(u)$ is -1 if $u \neq 1$ and 1 , otherwise.

By convention, $D_{i i}^{d}=0$.

It is easy to verify that for the case of identity permutation, the values do not change. Given range and bearing measurements, relative coordinates of the generated points for various operations with respect to the best vertex can be computed according to the following lemma. 


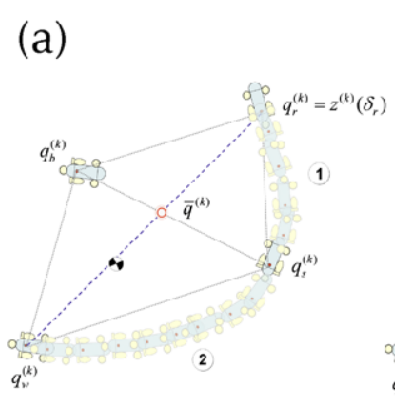

(c)
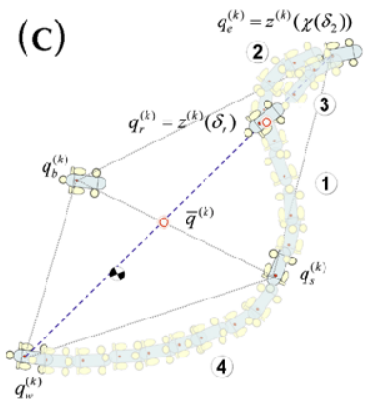

(d)

(b) $\quad a_{c}^{(k)}=z^{(k)}\left(x\left(\delta_{2}\right)\right)$
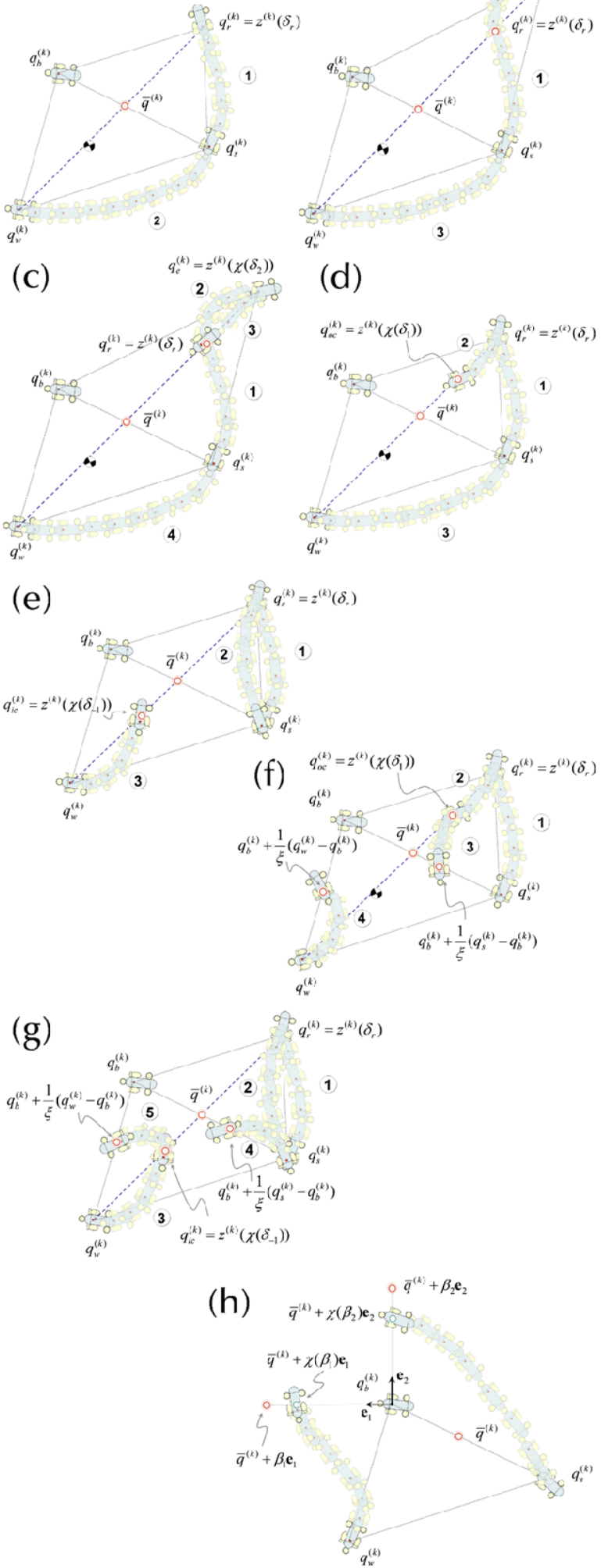

Fig. 8 Exploratory manoeuvres for enactment of the simplex steps. (a) Successful reflection. (b) Successful expansion. (c) Unsuccessful expansion. (d) Successful outside contraction. (e) Inside contraction. (f) Shrinking after unsuccessful outside contraction. (g) Shrinking after unsuccessful inside contraction. (h) Oriented restart
Lemma 6 For every $\delta$, the coordinates of a generated point in terms of range and bearing $\left(D_{\delta b}^{d}, \theta_{\delta b}^{d}\right)$ are given by

$$
\begin{aligned}
D_{\delta b}^{d} & =\sqrt{\left(\frac{1}{2} D_{s b}^{d}\right)^{2}+(\delta a)^{2}-2 D_{s b}^{d}|\delta a| \tilde{\vartheta}}, \\
\theta_{\delta b}^{d} & =\operatorname{acos}\left(\frac{v_{2} \cdot e_{1}}{\left\|v_{2}\right\|}\right)
\end{aligned}
$$

where

$$
\begin{aligned}
& a=\sqrt{\left(\frac{1}{2} D_{s b}^{d}\right)^{2}+\left(D_{w b}^{d}\right)^{2}-D_{s b}^{d} D_{w b}^{d} \tilde{a},} \\
& \tilde{a}=\left(\begin{array}{c}
\cos \left(\theta_{s b}^{d}\right) \\
\sin \left(\theta_{s b}^{d}\right)
\end{array}\right) \cdot\left(\begin{array}{c}
\cos \left(\theta_{w b}^{d}\right) \\
\sin \left(\theta_{w b}^{d}\right)
\end{array}\right), \\
& v_{2}=\frac{1}{2} D_{s b}^{d}\left(\begin{array}{c}
\cos \left(\theta_{s b}^{d}\right) \\
\sin \left(\theta_{s b}^{d}\right)
\end{array}\right)+\delta a\left(\begin{array}{c}
\cos (\vartheta) \\
\sin (\vartheta)
\end{array}\right), \\
& \tilde{\vartheta}=\operatorname{acos}\left(\left\langle-\left(\begin{array}{c}
\cos \left(\theta_{s b}^{d}\right) \\
\sin \left(\theta_{s b}^{d}\right.
\end{array}\right), \operatorname{sign}(\delta)\left(\begin{array}{c}
\cos (\vartheta) \\
\sin (\vartheta)
\end{array}\right)\right\rangle\right), \\
& \vartheta=\operatorname{acos}\left(\frac{v_{1} \cdot e_{1}}{\left\|v_{1}\right\|}\right), \\
& v_{1}=\frac{1}{2} D_{s b}^{d}\left(\begin{array}{c}
\cos \left(\theta_{s b}^{d}\right) \\
\sin \left(\theta_{s b}^{d}\right)
\end{array}\right)-D_{w b}^{d}\left(\begin{array}{c}
\cos \left(\theta_{w b}^{d}\right) \\
\sin \left(\theta_{w b}^{d}\right)
\end{array}\right) .
\end{aligned}
$$

Moreover, the centroid is given by $\left(\frac{1}{2} D_{s b}^{d}, \theta_{s b}^{d}\right)$, and the oriented restart points are given by

$$
\begin{aligned}
& \left(D_{o 1 b}^{d}, \theta_{o 1 b}^{d}\right) \\
& \quad=\left(\frac{1}{2} \sigma_{-}\left(S^{(k)}\right), \frac{1}{2}\left(\pi-\eta\left(\operatorname{sign}\left(\left\langle D\left(F: S^{(k)}\right), e_{1}\right\rangle\right)\right) \pi\right)\right)
\end{aligned}
$$

and

$$
\begin{aligned}
& \left(D_{o 2 b}^{d}, \theta_{o 2 b}^{d}\right) \\
& =\left(\frac{1}{2} \sigma_{-}\left(S^{(k)}\right),\right. \\
& \left.\quad \frac{\pi}{2}+\frac{1}{2}\left(\pi-\eta\left(\operatorname{sign}\left(\left\langle D\left(F: S^{(k)}\right), e_{2}\right\rangle\right)\right) \pi\right)\right) .
\end{aligned}
$$

Figure 9 illustrates some of the variables. Each step of the algorithm is executed by determining which of the worst and next best robots should go to which of the desired configurations designed above. Convergence to any of the desired locations signals the start of the next consecutive step. Note that the way we implement the algorithm makes sure that the shape of the simplex does not drift due to inaccuracies in reaching the desired points exactly. This is because 


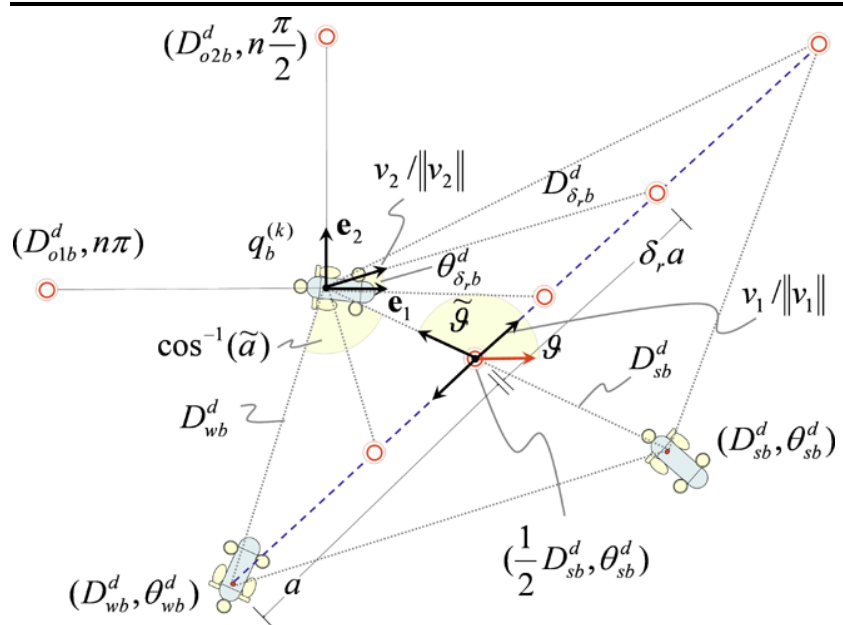

Fig. 9 The variables used in the calculation of the relative coordinates of the generated points with respect to the pivot robot

$\left(D_{21}^{d}, \theta_{21}^{d}\right)$ and $\left(D_{31}^{d}, \theta_{31}^{d}\right)$ are determined by $\left(D_{\delta b}^{d}, \theta_{\delta b}^{d}\right)$ or $\left(D_{o j b}^{d}, \theta_{o j b}^{d}\right), j \in\{1,2\}$, which do not depend on the actual range and bearing relations between the robots. The only drift is positional. A nonholonomic control rule can be used for ordinary operations. For shrink and oriented restart operations, local avoidance strategies should be used as the path of the moving robots may get very close to the pivot robot. After the completion of the steps, the next synchronization point $t_{1}$ triggers the second round.

Unlike the algorithm, where decisions are made instantaneously, a finite amount of time is elapsed before the completion of a round, during which the formation may be perturbed. For the algorithm to work, certain rather mild conditions have to be satisfied.

Definition 4 Given the simplex $S^{(k)}\left[q_{b}^{(k)}, q_{s}^{(k)}, q_{w}^{(k)}\right]$, corresponding to iterate of the simplex algorithm, the vector

$$
\begin{aligned}
& \left(q_{b}^{(k)}, q_{s}^{(k)}, q_{w}^{(k)}, z^{(k)}\left(\delta_{r}\right), z^{(k)}\left(\chi\left(\delta_{1}\right)\right),\right. \\
& \left.z^{(k)}\left(\chi\left(\delta_{2}\right)\right), z^{(k)}\left(\chi\left(\delta_{-1}\right)\right)\right)
\end{aligned}
$$

is called the augmented simplex and is denoted by $\tilde{S}^{(k)}$. The components are denoted $\tilde{S}_{i}^{(k)}, i=0, \ldots, 5$.

Definition 5 An $\varepsilon$-neighborhood of an augmented simplex $\tilde{S}^{(k)}$ is defined by

$B_{\varepsilon}\left(\tilde{S}^{(k)}\right)=\prod_{i=0}^{5} B_{\varepsilon}\left(\tilde{S}_{i}^{(k)}\right)$,

where it is assumed that

$$
\bigcap_{i=0}^{5} B_{\varepsilon}\left(\tilde{S}_{i}^{(k)}\right)=\varnothing .
$$

Definition 6 We say that a vector $S$ is $\varepsilon$-equivalent to $\tilde{S}^{(k)}$ and denote it by $S \sim \tilde{S}^{(k)}$ if $S \in B_{\varepsilon}\left(\tilde{S}^{(k)}\right)$, and, for every $i$, $S_{i} \in B_{\varepsilon}\left(\tilde{S}_{i}^{(k)}\right)$.

Definition 7 Suppose that, for each $i, j=0, \ldots, 5, i \neq j$, the relation

$\left(F\left(\tilde{S}_{i}^{(k)}\right), \mu_{g}\left(\tilde{S}_{i}^{(k)}\right)\right)\left[\tau_{\mu}, r_{\alpha}^{i j}\right]\left(F\left(\tilde{S}_{j}^{(k)}\right), \mu_{g}\left(\tilde{S}_{j}^{(k)}\right)\right)$

is satisfied, where $r^{i j} \in\{<,>, \leq, \geq\}$. We call $\tilde{S}^{(k)}$ invariant under an $\varepsilon$-perturbation if, for every $S \sim \tilde{S}^{(k)}$, we have that

$\left(F\left(S_{i}\right), \mu_{g}\left(S_{i}\right)\right)\left[\tau_{\mu}, r_{\alpha}^{i j}\right]\left(F\left(S_{j}\right), \mu_{g}\left(S_{j}\right)\right)$,

for every $i$ and $j, i \neq j$.

Note that invariance is actually a feature of the environment. If the environment or its measurements are not static, a new definition is required.

Definition 8 If $t_{0}$ and $t_{f}$ mark the start and end times of a single run of the inner loop in the algorithm, then $\tilde{S}^{(k)}\left(t_{0}\right)$ is called temporally invariant under $\varepsilon$-perturbation if, for every $S \sim \tilde{S}^{(k)}\left(t_{0}\right)$ and every $t \in\left[t_{0}, t_{f}\right]$, we have

$\left(F\left(S_{i}(t)\right), \mu_{g}\left(S_{i}(t)\right)\right)\left[\tau_{\mu}, r_{\alpha}^{i j}\right]\left(F\left(S_{j}(t)\right), \mu_{g}\left(S_{j}(t)\right)\right)$.

These conditions may or may not be severe restrictions, depending on the application. If the size of the simplex is sufficiently large, it should not be a problem.

System discrete automaton: The whole system can be modelled by a hybrid automaton which is executed by an imaginary formation controller which manages the operations of vehicles in the formation. The discrete part of the automaton mimics the modified simplex algorithm, while the continuous part describes the manoeuvres. Figure 10 shows this hybrid automaton. For the sake of clarity, the notation has been simplified.

The horizontal motion of the robot $\boldsymbol{R}_{i}$ is denoted by $\left(\dot{x}_{i}(t), \dot{y}_{i}(t)\right)$ which is the result of the horizontal controller $\boldsymbol{C}_{h}$ producing torques $\left(\tau_{b r}^{h}, \tau_{b l}^{h}\right)$ (for the two left and right horizontal thrusters) to make sure that $D_{i p}(t) \rightarrow D_{i p}^{d}$ and $\theta_{i p}(t) \rightarrow \theta_{i p}^{d}$, where $\boldsymbol{R}_{p}$ is the pivot robot, $D_{i p}^{d}$ and $\theta_{i p}^{d}$ are desired range and bearing relative to the pivot robot, and $D_{i p}(t)$ and $\theta_{i p}(t)$ are current values of the range and bearing. We use the notation $\dot{q}_{i}(t)=\boldsymbol{C}_{h}\left(D_{i p}^{d}, \theta_{i p}^{d}\right)$ to represent the motion of a vehicle. The case $i=p$ is denoted $\dot{q}_{p}(t)=$ $\boldsymbol{C}_{h}(0,0)$ and corresponds to station keeping. The vertical motion is, likewise, denoted by $\dot{z}_{i}(t)=\boldsymbol{C}_{v}\left(\boldsymbol{D}^{d}\right)$, where the 
Fig. 10 Hybrid state automaton of the virtual formation controller. Diamonds and pentagons represent decision points. The black circle indicates the initial state. Numbers in circles represent priority of decisions

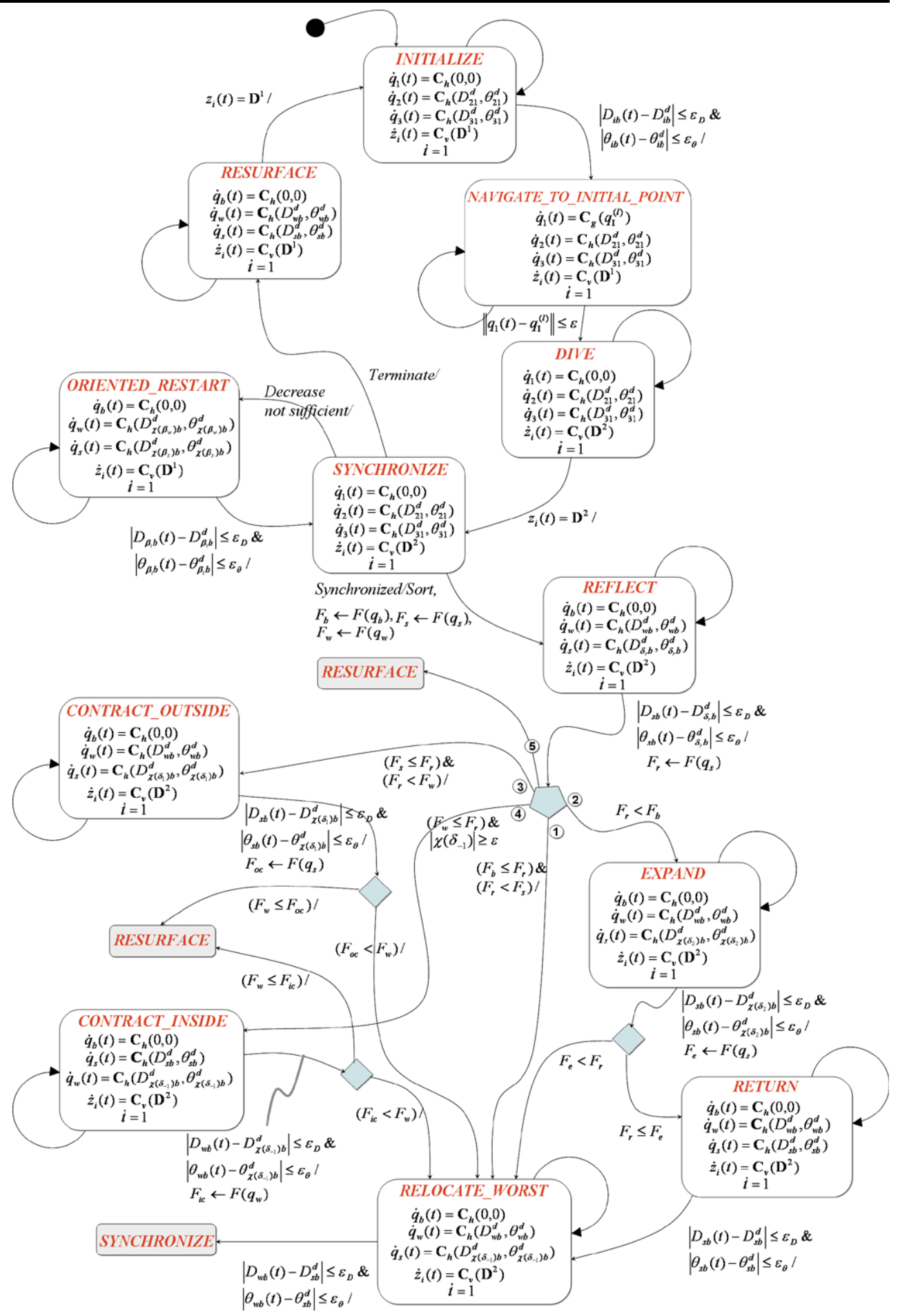

vertical controller $\boldsymbol{C}_{v}$ produces torques $\left(\tau_{f r}^{v}, \tau_{f l}^{v}, \tau_{b}^{v}\right)$ (for the two vertical thrusters on the sides and one at the aft) to assure the convergence of $D_{i}(t)$ (the depth measured by $\boldsymbol{R}_{i}$ ) to the desired depth $\boldsymbol{D}^{d}$. We distinguish between two depth values. $\boldsymbol{D}^{1}$ is the depth used for resurfacing (near the sur- face) and $\boldsymbol{D}^{2}$ which is the nominal depth of the operating plane. The horizontal control scheme $\dot{q}_{p}(t)=\boldsymbol{C}_{g}\left(q^{d}\right)$ navigates $\boldsymbol{R}_{p}$ to location $q^{d}$. It is used to relocate the formation to a new initial point determined by the probabilistic restart strategy. 
Fig. 11 Plots. (a) Inter-robot distances: (solid) $\left\|q_{0}-q_{1}\right\|$, (dot) $\left\|q_{0}-q_{2}\right\|,($ dash $)$ $\left\|q_{1}-q_{2}\right\|$. (b) Diameters: (solid) $\operatorname{diam}_{i}(S),($ dot $)$ $\operatorname{diam}_{o}(S)$. (c) Field values: (solid) $F\left(q_{0}\right)$, (dot) $F\left(q_{1}\right)$, (dash) $F\left(q_{2}\right)$. (d) Angles between edges: (solid) $\varphi_{0,12}$, (dash) $\varphi_{1,02}$, (dot) $\varphi_{2,01}$. (e) $v(S)$
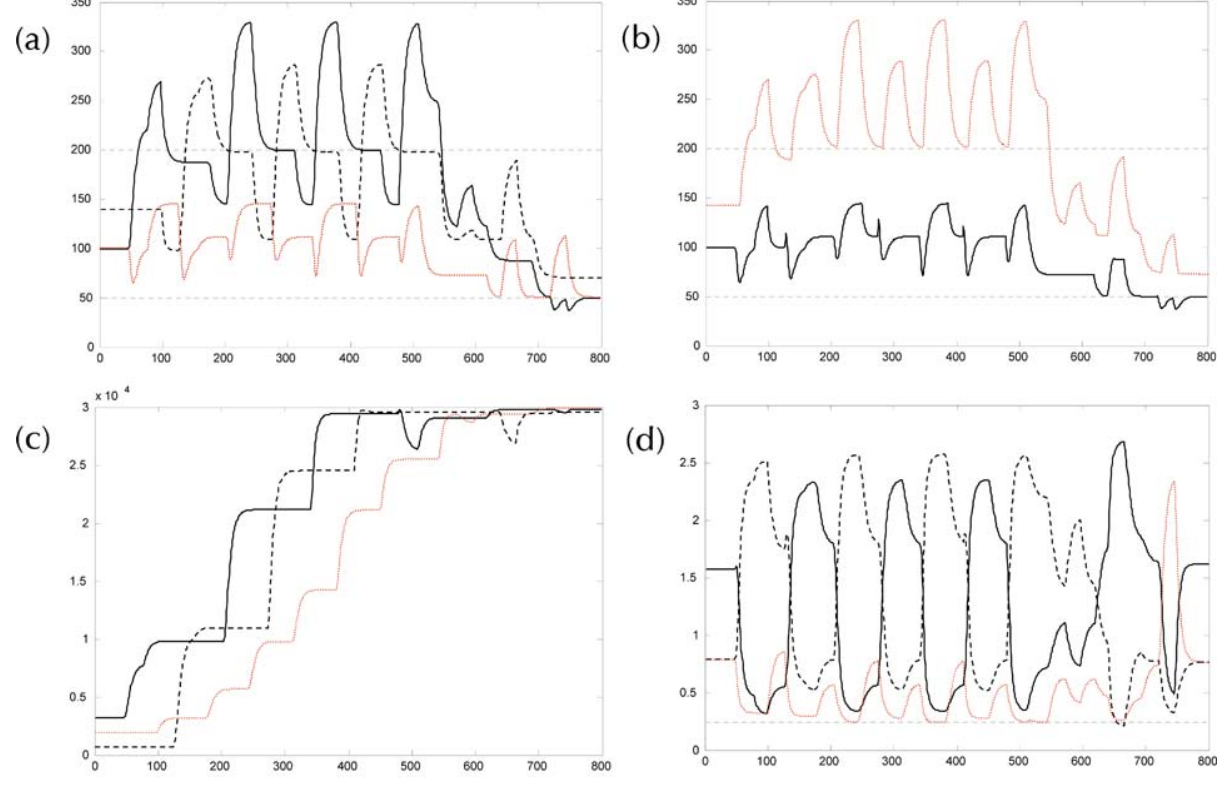

(d)

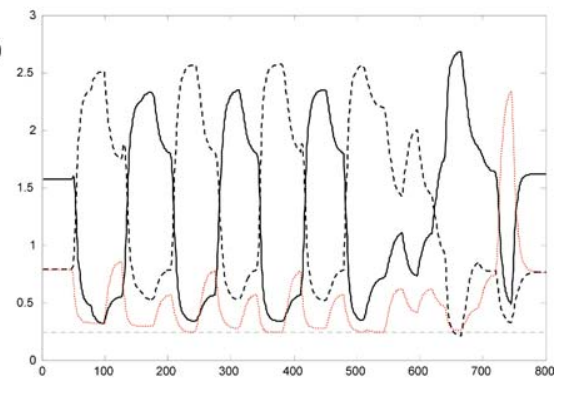

(e)

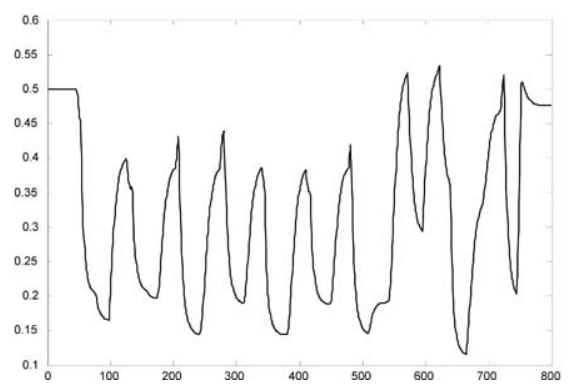

\section{Simulations}

In this section, we present a few simulation runs which are supposed to show the behaviour of the modified NelderMead algorithm.

Figure 12(a) shows localization of a local minimum by a formation using only reflection (basic simplex method). Figure 12(b) shows the trace of a formation climbing a noisy field. Figure 12(c) shows a scenario where motion is constrained to a part of the field due to the barrier shown as a line. Figure 12(d) shows the situation where the optimum of the field is located very close to the boundary. The formation goes through two oriented restarts and finally terminates operation as it has to shrink.

Figure 11 shows plots of inter-robot distances, triangle diameters, field values, angles, and finally $v(S)$, for a simulation run with $D_{l}=50, D_{u}=200$, and $\varphi_{l}=\pi / 10$.

\section{Conclusions and future research}

In this paper, we examined the possibility of using the Nelder-Mead extension to the basic simplex method for lo- calizing optima of natural fields by networks of autonomous robots playing the role of sample points. This problem corresponds to nonlinear function minimization or maximization in optimization theory. The proposed strategy is only one of many direct and/or derivative-free search methods proposed in mathematical literature in the past. Close examination of these methods (when implemented by a robotic network) reveals certain similarities: (1) the robot aggregate is deformable, (2) it moves from one iteration to the next as opposed to continuous motion in rigid formations, (3) in each iterate, some of the robots remain stationary while others carry on exploratory motions to go to sample points in the vicinity of the aggregate, (4) relative values at sampled points are used as a basis for determining the next iterate. It would certainly be worthwhile to look into general frameworks for such exploratory aggregates (see Kalantar 2007 for such an attempt). Future research should address: (1) study of faithfulness of a robotic implementation to the original algorithm in the face of uncertainties, (2) the challenge of station keeping in underwater environments, (3) exploring other similar search strategies, (4) designing a general system which can autonomously implement a given 
(a)

(b)

(c)

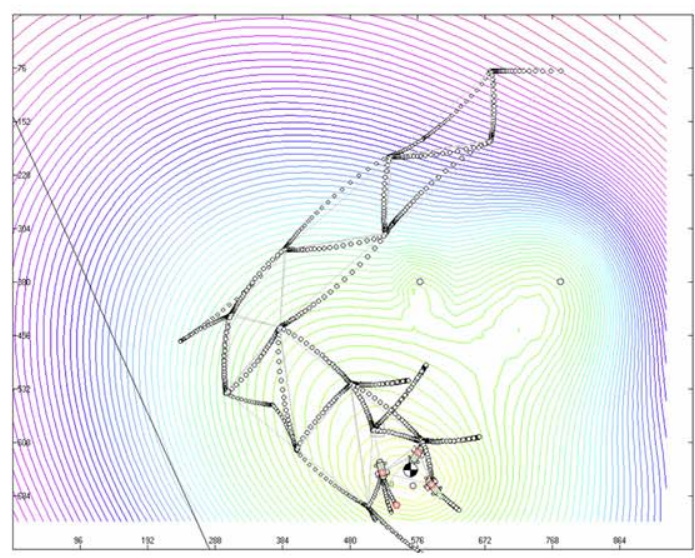

(d)

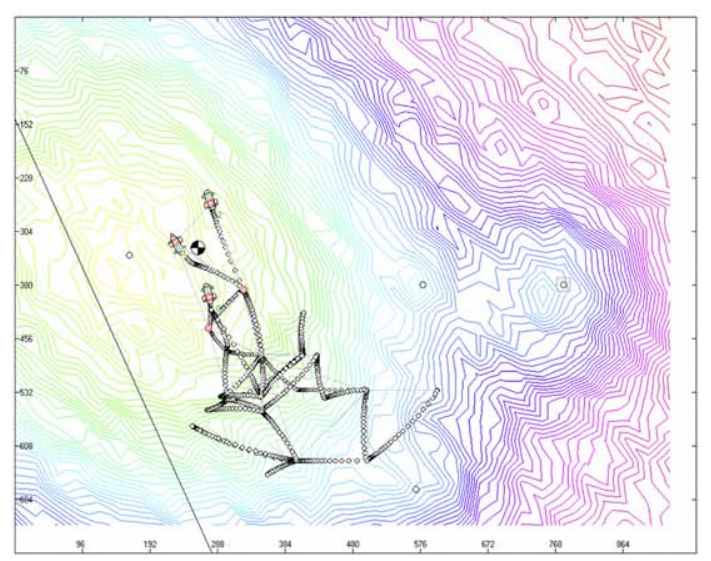

Fig. 12 (a) Basic simplex. (b) Noisy field. (c) Constrained region. (d) Optimum close to barrier; the formation has to shrink blueprint for a search algorithm, (5) peculiarities of timevarying fields, (6) communication and synchronization issues.

\section{References}

Burian, E., Yoerger, D., Bradley, A., \& Singh, H. (1996). Gradient search with autonomous underwater vehicles using scalar measurements. In Proceedings of IEEE symposium on autonomous underwater vehicle technology, Monterey, CA, USA.

Byrne, R. H., Eskridge, S. E., Hurtado, J. H., \& Savage, E. L. (2003). Algorithms and analysis for underwater vehicle plume tracing (SANDIA Report SAND2003-2643). Sandia National Laboratories.

Conn, A. R., Scheinberg, K., \& Toint, P. L. (1997). On the convergence of derivative-free methods for unconstrained optimization. In $A p$ proximation theory and optimization: tributes to M. J. D. Powell. Cambridge: Cambridge University Press.

de Sousa, J. B., Johansson, K. H., Speranzon, A., \& Silva, J. (2005). A control architecture for multiple submarines in coordinated search missions. In Proceedings of the 16th IFAC world congress.

de Sousa, J. B., Johansson, K. H., Silva, J., \& Speranzon, A. (2006). A verified hierarchical control architecture for coordinated multivehicle operations. International Journal of Adaptive Control and Signal Processing.

Eren, T. (2004). Rigid formations of autonomous agents. $\mathrm{PhD}$ thesis, Yale University New Haven, CT, USA, order number: AAI3125188.

Haftka, R. T., \& Guerdal, Z. (1993). Elements of structural optimization (3rd ed.). Boston: Kluwer Academic.

Kalantar, S. (2007). Formations of small submersible robots and autonomous mapping of underwater phenomena. $\mathrm{PhD}$ thesis, Department of Information Engineering, The Australian National University.

Kalantar, S., \& Zimmer, U. (2007a). Simplex formations of autonomous vehicles and adaptive sampling strategies. In Proceedings of TAROS, Aberystwyth, UK.

Kalantar, S., \& Zimmer, U. (2007b). Adaptive iso-altitude tracking and terrain reconstruction by submarine vehicle formations. In Proceedings of ACRA, Brisbane, Australia.

Kelley, C. T. (1999). Detection and remediation of stagnation in the Nelder-Mead algorithm using a sufficient decrease condition. SIAM Journal of Optimization, 10(1).

Kolda, T. G., Lewis, R. M., \& Torczon, V. (2003). Optimization by direct search: new perspectives on some classical and modern methods. SIAM Review, 45(3).

Lagarias, J. C., Reeds, J. A., Wright, M. H., \& Wright, P. E. (1998). Convergence properties of the Nelder-Mead simplex method in low dimensions. SIAM Journal of Optimization, 9(1).

Lewis, R. M., Torczon, V., \& Trosset, M. W. (2000). Direct search methods: then and now (Technical Report 2000-26). Institute for Computer Applications in Science and Engineering, NASA.

Luersen, M. A., \& Le Riche, R. (2004). Globalized Nelder-Mead method for engineering optimization. Computers and Structures, 82.

Luersen, M. A., Le Riche, R., \& Guyon, F. (2004). A constrained, globalized, and bounded Nelder-Mead method for engineering optimization. Structural and Multidisciplinary Optimization, 27.

McKinnon, K. I. M. (1998). Convergence of the Nelder-Mead simplex method to a nonstationary point. SIAM Journal of Optimization, 9(1). 
Nazareth, L., \& Tseng, P. (2002). Gilding the lily: a variant of the Nelder-Mead algorithm. Computational Optimization and Applications, 22(1).

Nelder, J. A., \& Mead, R. (1965). A simplex method for function minimization. The Computer Journal, 7.

Ögren, P., Fiorelli, E., \& Leonard, N. E. (2002). Formations with a mission: stable coordination of vehicle group maneuvers. In Proceedings of the 15th international symposium on mathematical theory of networks and systems.

Ögren, P., Fiorelli, E., \& Leonard, N. E. (2004). Cooperative control of mobile sensor networks: adaptive gradient climbing in a distributed environment. IEEE Transactions on Automatic Control, 49(8).

Pang, S., Farrell, J. A., Arrieta, R. M., \& Li, W. (2003). AUV reactive planning: deepest point. In Proceedings of IEEE oceans conference.

Robinet III, R. D. (2004). Stability and control of collective systems. Journal of Intelligent and Robotic Systems, 39.

Silva, J., Speranzon, A., de Sousa, J. B., \& Johansson, K. H. (2004). Hierarchical search strategy for a team of autonomous vehicles. In Proceedings of the 5th IFAC symposium on intelligent autonomous vehicles.

Speranzon, A., Silva, K. H., de Sousa, J. B., \& Johansson, K. H. (2004). On collaborative optimization and communication for a team of autonomous underwater vehicles. In Proceedings of Reglermöte.

Takahama, T., \& Sakai, S. (2005). Constrained optimization by applying the constrained method to the nonlinear simplex method with mutations. IEEE Transactions on Evolutionary Computation, 9(5).

Wright, M. H. (1996). Direct search methods: once acorned, now respectable. In Proceedings of Dundee biennial conference on numerical analysis. Addison-Wesley: Longman.

Zhang, F., \& Leonard, N. E. (2005). Generating contour plots using multiple sensor platforms. In Proceedings of IEEE swarm intelligence symposium

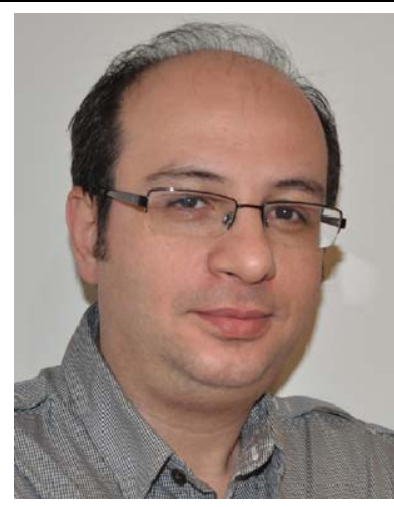

Shahab Kalantar got his Master's degree in Robotics and Machine Intelligence in 1997 from Tehran University and his Bachelor's in Applied Mathematics in 1991. He received his $\mathrm{PhD}$ degree at the Australian National University in 2007. His current research interest is the interaction of deformable formations of autonomous vehicles with environmental fields.

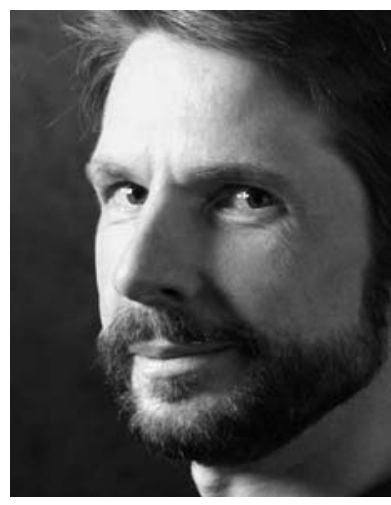

Uwe R. Zimmer is currently a fellow at the Australian National University at the Faculty of Engineering and Information Technology and at the Research School of Information Sciences and Engineering. He received his Dr. rer. nat. degree in 1995 at the University of Kaiserslautern, Germany. Building up an underwater research group in Germany at the former German National Research Center for Information Technology, and setting up a research laboratory for autonomous robust systems in Kyushu, Japan have been the other major activities since then. He is currently the head of the underwater robotics group at the Australian National University. His major research interests are in distributed swarm control and communication with application to real-time and real-world environments. 